\title{
Nonconformality, subregion complexity, and meson binding
}

\author{
Mahsa Lezgi, ${ }^{1, *}$ Mohammad Ali-Akbari, ${ }^{1, \dagger}$ and Mohammad Asadi $\odot^{2, *}$ \\ ${ }^{1}$ Department of Physics, Shahid Beheshti University, 1983969411, Tehran, Iran \\ ${ }^{2}$ School of Particles and Accelerators, Institute for Research in Fundamental Science (IPM), \\ P.O. Box 19395-5746, Tehran, Iran
}

(Received 7 March 2021; accepted 7 June 2021; published 2 July 2021)

\begin{abstract}
We study holographically the zero and finite temperature behavior of the potential energy and holographic subregion complexity corresponding to a probe meson in a nonconformal model. Interestingly, in the specific regime of the model parameters, at zero and low temperatures, we find a nicely linear relation between dimensionless meson potential energy and dimensionless volume implying that the less binding meson state needs less information to be specified and vice versa, but this behavior can not be confirmed in the high temperature limit. We also observe that the nonconformal corrections increase the holographic subregion complexity in both the zero and finite temperature states. However, nonconformality has a decreasing effect on the dimensionless meson potential energy. We finally find that in the vicinity of the phase transition, the zero temperature meson state is more favorable than the finite temperature state, from the holographic subregion complexity point of view.
\end{abstract}

DOI: 10.1103/PhysRevD.104.026001

\section{INTRODUCTION}

The gauge/gravity duality is a conjectured relationship between quantum field theory and gravity. This duality is a strong-weak duality which maps a strongly-coupled quantum gauge field theory to a weakly-coupled classical gravity in a higher dimension [1]. The duality provides an important framework to study key properties of the boundary field theory dual to some gravitational theory on the bulk side. This idea is used to describe phenomena in strongly-coupled quantum field theories ranging from condensed matter physics to low-energy quantum chromodynamics (QCD), the theory of the strong interactions [2]. The most significant example of gauge/gravity duality is the AdS/CFT correspondence which proposes a duality between IIB string theory on anti-de Sitter (AdS) spacetimes in $d+1$ dimensions and $d$-dimensional superconformal field theories. This framework has been applied to study quantities such as Wilson loops, entanglement entropy and has recently been extended to the quantum computational complexity in field theory. The generalization of AdS/CFT correspondence to field theories which

\footnotetext{
*_lezgi@sbu.ac.ir

m_aliakbari@sbu.ac.ir

\#m_asadi@ipm.ir
}

Published by the American Physical Society under the terms of the Creative Commons Attribution 4.0 International license. Further distribution of this work must maintain attribution to the author(s) and the published article's title, journal citation, and DOI. Funded by SCOAP ${ }^{3}$. are not conformal seems to be important. It is then interesting to develop our understanding of this duality for more general cases.

There are many different families of nonconformal field theories and one can study the effect of the noncoformality on their physical observables [3]. Some of the most important gauge-invariant and nonlocal observables in gauge theories are Wilson loops. In quantum field theory, potential energy can be obtained by this quantity as a gauge invariant nonlocal operator. The expectation value of Wilson loops has valuable applications to the confinement/deconfinement and QCD-like theories [4-7]. In particular the expectation value of this operator on a rectangular loop gives us the potential energy between a static quark and antiquark.

Entanglement entropy is another intriguing nonlocal quantity which measures the quantum entanglement between two subsystems of a given system. This quantity is extremely useful in many quantum systems, ranging from condensed matter physics to black hole physics. It can be used to classify the various quantum phase transitions and critical points [8-13]. Since the quantum field theories have infinite degrees of freedom, the entanglement entropy is divergent. Thus, it is a scheme-dependent quantity and needs to be regulated. It has been shown that the leading divergence term is proportional to the area of the entangling surface (for $d>2$ ) [14,15]. The entanglement entropy has a holographic dual given by the area of minimal surface extended into the bulk whose boundary coincides with the boundary of the subregion [16].

Concepts from quantum information theory are having a rapidly growing influence in investigations of quantum field 
theory and quantum gravity. One of the most important information quantities is quantum computational complexity. This is a state-dependent quantity which measures how difficult it is to prepare a given state, from the reference state. More precisely, quantum computational complexity involves minimizing the number of unitary transformations required to transform a reference state to a target state. For more details, see $[17,18]$. There have been two prescriptions to calculate the quantum complexity in terms of the gauge/ gravity duality, which are known as the Complexity = Volume (CV) conjecture [19] and the Complexity = Action (CA) conjecture [20,21]. Note that these proposals correspond to the complexity of a pure state in the whole boundary space of the dual quantum field theory. If one would like to compute the complexity of a mixed state, corresponding to a subsystem, then one should generalize the notion of complexity to the subregion complexity. There are two known proposals called holographic subregion complexity (HSC) for the CV conjecture [22] and the CA conjecture [23]. The reader can find other studies on the holographic subregion complexity in [24-30].

The remainder of the paper is organized as follows: Section II considers a brief review of modified $\mathrm{AdS}_{5}$ (MAdS) background and its black hole version, which is called modified black hole (MBH), and we investigate the thermodynamic properties of them including entropy, density, and pressure, which are given in terms of nonconformal parameter theory. In Sec. III, we study some nonlocal observables such as potential energy, entanglement entropy, and HSC corresponding to a bound state meson and express how to calculate them through holographic prescriptions. In Sec. IV we study the meson potential energy at both zero and finite temperatures. In order to obtain the analytical results we develop a systematic expansion (see [31-33]), up to some specific order of the expansion parameter and consider a specific limit of the underlying field theory called high energy limit. However, since we cannot obtain analytical results, we will not study the low energy limit. At finite temperature, we focus on two favorable low and high temperature limits. In Sec. V, we go through the same steps as Sec. IV and obtain the analytical results corresponding to the HSC. At the end of this section we reach an interesting result regarding a linear relation between dimensionless HSC and dimensionless meson potential energy. In Sec. VI, we conclude with a brief discussion of our results and we consider some directions for future research. In Appendix A, we present the full details of our calculations corresponding to meson potential energy. In Appendix B we do the same for HSC results.

\section{REVIEW ON THE BACKGROUNDS}

We are interested in studying the nonlocal quantities such as Wilson loops and HSC of a probe meson (a stable quark and antiquark bound state) using the framework of holography. Therefore, we start with a five-dimensional background and its black hole version, which is called modified $\mathrm{AdS}_{5}$ and modified black hole, respectively. These backgrounds are dual to QCD-like gauge theories at zero and nonzero temperature.

\section{A. The backgrounds}

The gravitational backgrounds we study here are the following MAdS background [34]

$d s^{2}=\frac{r^{2}}{R^{2}} g(r)\left(-d t^{2}+d \vec{x}^{2}+\frac{R^{4}}{r^{4}} d r^{2}\right), \quad g(r)=e^{\frac{c R^{4}}{2 r^{2}}}$,

and the black hole version of the above model, the $\mathrm{MBH}$ background [35]

$$
\begin{aligned}
& d s^{2}=\frac{r^{2}}{R^{2}} g(r)\left(-f(r) d t^{2}+d \vec{x}^{2}+\frac{R^{4}}{r^{4} f(r)} d r^{2}\right), \\
& f(r)=1-\frac{r_{h}^{4}}{r^{4}},
\end{aligned}
$$

where $r$ is the radial coordinate, $r_{h}$ is the position of the horizon, $\vec{x} \equiv(x, y, z), R$ is the asymptotic $\mathrm{AdS}_{5}$ radius and the boundary is located at $r=\infty$ where the QCD-like model lives. The modifier parameter $c$ has (energy) ${ }^{2}$ dimension whose value is fixed from the $\rho$ meson trajectory estimated to be of order $0.9 \mathrm{GeV}^{2}$ [35]. There is an upper bound on the maximum value of the radial coordinate called $r_{c}=R^{2} \sqrt{\frac{c}{2}}$. Note that as $c$ goes to zero then $r$ is not bounded, as expected for the AdS background [34]. In the QCD-like theory, $r_{c}$ corresponds to a mass gap which is the lowest excitation and its energy order is the same as the QCD scale. Therefore, we assign a energy scales $\Lambda_{c}$ to $r_{c}$ in such a way that $\Lambda_{c} \equiv \sqrt{c}$. The asymptotically $\mathrm{AdS}_{5}$ solution and its black hole version will be respectively recovered if one sets the modifier parameter zero, say $c=0$, in backgrounds (1) and (2).

\section{B. Thermodynamics}

Consider a general class of black brane metrics of the form

$$
d s^{2}=g(r)\left[-f(r) d t^{2}+d \vec{x}^{2}\right]+\frac{1}{h(r)} d r^{2},
$$

where $f(r)$ and $h(r)$ have a first order zero at the horizon $r=r_{h}$, while $g(r)$ is nonvanishing there. The Hawking temperature $T$ and the entropy density $s$ of the black brane is given by

$$
T=\left.\frac{\sqrt{g(r) f^{\prime}(r) h^{\prime}(r)}}{4 \pi}\right|_{r=r_{h}}, \quad s=\left.\frac{g(r)^{\frac{3}{2}}}{4 G_{N}}\right|_{r=r_{h}},
$$

where $G_{N}$ is the Newton constant. According to (2) and using the fact that $f\left(r_{h}\right)=0$, the temperature and the entropy density of the boundary field theory become 


$$
T=\frac{r_{h}}{R^{2} \pi}, \quad s=\frac{r_{h}^{3} e^{\frac{3 \Lambda_{\Lambda}^{2} R^{4}}{4 r_{h}^{2}}}}{R^{3} 4 G_{N}} .
$$

It is clearly seen from the above equations that the theory will be conformal if one takes the limit $c \rightarrow 0$ ( or $\Lambda_{c} \rightarrow 0$ ). Hence, one expects that the entropy density must coincide with that of a relativistic conformal theory which scales as $T^{3}$. We are now in a position to determine the pressure $p$ and the energy density $\epsilon$ of the thermal system through standard thermodynamic relations

$$
p=\int d T s(T), \quad \varepsilon+p=T s .
$$

Using (2), (5), and (6) the pressure and the energy density are expressed

$p=\frac{\pi^{3} R^{3}}{4 G_{N}}\left[\frac{3 \Lambda_{c}^{2}}{4 \pi^{2} T^{2}}\left(\frac{3 \Lambda_{c}^{2} T^{2}}{16 \pi^{2}}+\frac{T^{4}}{4}\right)-\frac{9 \Lambda_{c}^{4} E i\left(\frac{3 \Lambda_{c}^{2}}{4 \pi^{2} T^{2}}\right)}{64 \pi^{4}}\right]$,

$\varepsilon=\frac{\pi^{3} R^{3}}{4 G_{N}}\left[e^{\frac{3 \Lambda_{c}^{2}}{4 \pi^{2} T^{2}}}\left(\frac{3 T^{4}}{4}-\frac{3 \Lambda_{c}^{2} T^{2}}{16 \pi^{2}}\right)+\left(\frac{9 \Lambda_{c}^{4} E i\left(\frac{3 \Lambda_{c}^{2}}{4 \pi^{2} T^{2}}\right)}{64 \pi^{4}}\right)\right]$,

where $\operatorname{Ei}(x)$ is the exponential integral defined for a real nonzero values of $x$

$$
E i(x)=-\int_{-x}^{\infty} d t \frac{e^{-t}}{t} .
$$

\section{WILSON LOOP, ENTANGLEMENT ENTROPY AND SUBREGION COMPLEXITY}

We explore the behavior of some nonlocal observables including potential energy and subregion complexity of a probe meson. To obtain analytic expressions for these observables we do a systematic expansion at both zero and finite temperature using the holography idea.

\section{A. Wilson loop}

In quantum filled theory, the potential energy can be obtained by the Wilson loop operator. The expectation value of this operator on a rectangular loop, $\mathcal{R}$ with two sides, time $\tau$ and distance $l$, where $\tau \gg l$, gives us the potential energy between a static quark and antiquark with the distance $l$. The holographic prescription of the expectation value of the Wilson loop is the on-shell action of a classical string $S(\mathcal{R})$ whose endpoints correspond to a quark and antiquark on the boundary and is suspended from the boundary to the extra dimension in the bulk [36]

$$
\langle W(\mathcal{R})\rangle=e^{i S(\mathcal{R})} .
$$

It is then straightforward to find the potential energy corresponding to the binding energy of the meson.
Further information about the holographic Wilson loop can be found in [7].

\section{B. Entanglement entropy}

If we decompose the total Hilbert space of a system, $\mathcal{H}_{\text {tot }}$, into two subsystems $\mathcal{H}_{A}$ and $\mathcal{H}_{B}$ such that $\mathcal{H}_{\text {tot }}=\mathcal{H}_{A} \otimes \mathcal{H}_{B}$, then we will trace out the subsystem $B$ and define the reduced density matrix $\rho_{A}$ for the subsystem $A$ as $\rho_{A}=$ $\operatorname{Tr}_{B} \rho$ where $\rho$ is the total density matrix. The entanglement entropy $S_{A}$ measures how much information is hidden inside the subsystem $A$ and defined as the von Neumann entropy of the reduced density matrix $\rho_{A}$

$$
S_{A}=-\operatorname{tr} \rho_{A} \log \rho_{A} .
$$

The AdS/CFT correspondence provides an elegant way to compute the entanglement entropy in terms of a geometrical quantity on the bulk. This is the so-called holographic entanglement entropy formula, first proposed by Ryu and Takayanagi $[16,37]$

$$
S_{A}=\frac{\operatorname{Area}\left(\gamma_{A}\right)}{4 G_{N}^{d+2}},
$$

where $S_{A}$ is the holographic entanglement entropy for the sub-system $A, \gamma_{A}$ is a codimension-two minimal area surface (RT surface) whose boundary $\partial \gamma_{A}$ coincides with $\partial A$, and $G_{N}^{d+2}$ is the $d+2$-dimensional Newton constant.

\section{Subregion complexity: CV duality}

If we would like to compute the complexity of the mixed state, then we will need to extend the holographic complexity to the subregions. The holographic volume prescription for calculating subregion complexity states that the HSC for a subregion $A$ on the boundary equals the volume of codimension-one RT surface enclosed by $\gamma_{A}$ which is given by the following form [22]

$$
\mathcal{C}_{A}=\frac{V_{\gamma_{A}}}{8 \pi R G_{N}},
$$

where $R$ is the AdS radius and $\mathcal{C}_{A}$ is known as the HSC for the subregion $A$.

\section{POTENTIAL ENERGY}

Heavy-quark potential is one of the fundamental observables which is relevant to confinement. Based on [38] the following heavy-quark potential, Cornell potential, is proposed

$$
V(r)=-\frac{\kappa}{r}+\frac{r}{a^{2}}+\mathcal{V}
$$

where $\kappa$ is treated as a phenomenological parameter and $a$ is inferred from lattice gauge theory. These parameters are adjusted to be 
$\kappa \approx 0.48, \quad a \approx 2.34 \mathrm{GeV}^{-1}, \quad \mathcal{V} \approx-0.25 \mathrm{GeV}$.

The first term of the potential is in complete accordance with the known Coulomb potential at short distance $r \rightarrow 0$ and the second one with confinement at large distance $r \rightarrow \infty$.

At zero temperature, in the background (1), the potential energy between the quark and antiquark pair is obtained and shows that this background describes the low energy of QCD-like theory, confined phase [34]. This meson potential energy is given by

$$
V(r)= \begin{cases}p\left(-\frac{\kappa_{0}}{r}+\sigma_{0} r+O\left(r^{3}\right)\right) & r \rightarrow 0 \\ p(\sigma r) & r \rightarrow \infty,\end{cases}
$$

where $p \approx 0.94$ and $\kappa_{0} \approx 0.23$ are dimensionless parameters, and we have $\sigma_{0} \approx 0.16 \mathrm{GeV}^{2}$ and $\sigma \approx 0.19 \mathrm{GeV}^{2}$ for $c=0.9 \mathrm{GeV}^{2}$. These constants are fixed according to the Cornell potential (13). In the following subsections we would like to obtain analytically the potential energy of the probe meson at both zero and finite temperatures. In the rest of the paper we set the AdS radius $R=1$.

\section{A. Potential energy: zero temperature expansion}

In this section we consider a state which corresponds to the MAdS background (1) and probe this state with a meson in the QCD-like theory. On the gravity side, this meson is dual to a classical string whose dynamic is given by Nambu-Goto action

$$
S_{N G}=\frac{-1}{2 \pi \alpha^{\prime}} \int d \tau d \sigma \sqrt{-\operatorname{det}\left(g_{a b}\right)},
$$

where $\alpha^{\prime}=l_{s}^{2}$ in which $l_{s}$ is the string length and $g_{a b}$ is the induced metric on the world sheet. The world sheet can be parametrized by $\tau=t$ and $\sigma=x$ and by demanding that $\tau \rightarrow \infty$, its shape is given by $r(x)$. We set the quark and antiquark at $x=-l / 2$ and $x=l / 2$. At zero temperature using the MAdS background (1) we can easily see that

$$
S_{N G}=\frac{\tau}{\pi \alpha^{\prime}} \int_{0}^{\frac{l}{2}} \frac{c}{e^{2 r^{2}}} r \sqrt{\frac{r^{\prime 2}}{r^{2}}+r^{2}} d x
$$

where $r^{\prime}=d r / d x$. The above action is not explicitly dependent on $x$ so the corresponding Hamiltonian is a constant of motion. Hence, using the boundary condition $\left.r^{\prime}(x)\right|_{x=0}=0$, we get

$$
r^{\prime}(x)=\frac{r^{2}}{r_{*}^{2}} e^{\frac{-c}{2 r_{*}^{2}}} \sqrt{r^{4} e^{\frac{c}{r^{2}}}-r_{*}^{4} e^{\frac{c}{r_{*}^{2}}}}
$$

where $r_{*}=\left.r(x)\right|_{x=0}$ is the returning point of the string. By integration of Eq. (18) the characteristic length $l$ corresponding to the separation of quark and antiquark read as follows:

$$
\begin{aligned}
l\left(r_{*}\right) & =2 r_{*}^{2} e^{\frac{c}{2 r_{*}^{2}}} \int_{r_{*}}^{\infty} \frac{d r}{r^{2} \sqrt{r^{4} e^{\frac{c}{r^{2}}}-r_{*}^{4} e^{\frac{c}{r_{*}^{2}}}}} \\
& =\frac{2}{r_{*}} \int_{0}^{1} u^{2} e^{\left(\frac{r_{c}}{r_{*}}\right)^{2}\left(1-u^{2}\right)}\left(1-e^{2\left(\frac{r_{c}}{r_{*}}\right)^{2}\left(1-u^{2}\right)} u^{4}\right)^{-\frac{1}{2}} d u,
\end{aligned}
$$

where $u=r_{*} / r$. By substituting (18) in (17) and using [36] we have the following expression for the meson potential energy

$V_{q \bar{q}}\left(r_{*}\right)=\frac{r_{*}}{\pi \alpha^{\prime}} \int_{\delta}^{1} u^{-2} e^{\left(\frac{r_{c}}{r_{*}}\right)^{2} u^{2}}\left(1-e^{2\left(\frac{r_{c}}{r_{*}}\right)^{2}\left(1-u^{2}\right)} u^{4}\right)^{-\frac{1}{2}} d u$,

where $\delta$ is an ultra violet cutoff due to the UV divergence structure of the potential energy.

Unfortunately (20) can not be analytically solved. Therefore, we use binomial expansion, $(1-x)^{-\frac{1}{2}}=$ $\sum_{n=0}^{\infty} \frac{\Gamma\left(n+\frac{1}{2}\right)}{\sqrt{\pi} \Gamma(n+1)} x^{n},-1 \leq x<1$. By defining $x=e^{2\left(\frac{r_{c}}{r_{*}}\right)^{2}\left(1-u^{2}\right)} u^{4}$ and using the fact that $r_{c}<r_{*}$ one can easily see that $x<1$ and hence the sum is well defined. Following the above discussion, (19) and (20) can be indicated by the infinite series

$l\left(r_{*}\right)=\frac{2}{r_{*}} \sum_{n=0}^{\infty} \frac{\Gamma\left(n+\frac{1}{2}\right)}{\sqrt{\pi} \Gamma(n+1)} \int_{0}^{1} u^{4 n+2} e^{(2 n+1)\left(1-u^{2}\right)\left(\frac{r_{r}}{r_{*}}\right)^{2}} d u$,

$V_{q \bar{q}}\left(r_{*}\right)=\frac{r_{*}}{\pi \alpha^{\prime}} \sum_{n=0}^{\infty} \frac{\Gamma\left(n+\frac{1}{2}\right)}{\sqrt{\pi} \Gamma(n+1)} \int_{\delta}^{1} u^{4 n-2} e^{\left(2 n+(1-2 n) u^{2}\right)\left(\frac{r_{*}}{r_{*}}\right)^{2}} d u$.

In order to find the meson potential energy $V_{q \bar{q}}$ as a function of the characteristic length $l$ the following simple procedure is done. We should solve equation Eq. (21) for $r_{*}$ and then substitute it in Eq. (22) to obtain $V_{q \bar{q}}$ in terms of $l$. In practice, we can not analytically solve Eq. (21) to find $r_{*}$ as a function of $l$. Therefore, we need to focus on the specific limit, which we call the high energy limit. We will introduce this limit in the next part. Note that, in the low energy limit, i.e., $r_{*} \rightarrow r_{c}$ (or $l \Lambda_{c} \gg 1$ ), we do not reach the analytical results and then we have to neglect studying this limit.

\section{High energy limit}

As we mentioned at the end of the Sec. IVA, to find $V_{q \bar{q}}$ in terms of $l$ we focus on the high energy limit. On the gravity side, by this limit we mean that the upper bound on the maximum value of the radial coordinate $r_{c}$ should be much smaller than the turning point of the classical string $r_{*}$, i.e., $r_{c} \ll r_{*}$. On the field theory side, the energy scale which we assign to $r_{c}$, called $\Lambda_{c}$, should be much smaller than the energy scale corresponding to the probe meson, 
i.e., $l \Lambda_{c} \ll 1$. It is noticed that, in this limit, the corrections to the boundary observables are small and hence we can perturbatively do the calculations.

In high energy limit, from Eq. (21) and keeping up to the fourth order in $r_{c} / r_{*}$ we obtain

$l\left(r_{*}\right)=\frac{2}{r_{*}}\left[a_{1}+a_{2}\left(\frac{r_{c}}{r_{*}}\right)^{2}+a_{3}\left(\frac{r_{c}}{r_{*}}\right)^{4}\right], a_{1}, a_{2}, a_{3}>0$,

where numerical coefficients $a_{1}, a_{2}$ and $a_{3}$ are reported in Table I in Appendix A. Solving Eq. (23) perturabtively for $r_{*}$ and then considering the finite terms, the terms which do not include $\delta$ in the meson potential energy (the details of the calculations are written in Appendix A), we finally reach the following expression

$$
\begin{aligned}
\tilde{V}_{q \bar{q}}\left(l \Lambda_{c}\right) \equiv & \frac{\alpha^{\prime} V_{q \bar{q}}\left(l, l \Lambda_{c}\right)}{\Lambda_{c}} \\
= & \frac{1}{l \Lambda_{c}}\left(\bar{b}_{1}+\frac{\bar{b}_{2}}{2}\left(l \Lambda_{c}\right)^{2}+\frac{\bar{b}_{3}}{4}\left(l \Lambda_{c}\right)^{4}\right), \\
& \bar{b}_{1}<0, \quad \bar{b}_{2}, \bar{b}_{3}>0,
\end{aligned}
$$

where $\tilde{V}_{q \bar{q}}$ is the dimensionless meson potential energy at zero temperature and numerical coefficients $\bar{b}_{1}, \bar{b}_{2}$, and $\bar{b}_{3}$ are shown in Table I in Appendix A. Since the underlying field theory is conformal, it is expected that the dimensionless parameter $l \Lambda_{c}$ will appear. To correctly compare the underlying field theory with that which includes the energy scale $\Lambda_{c}$, we make the meson potential energy dimensionless. By doing so we can get meaningful and intuitive results by taking the conformal limit $l \Lambda_{c} \rightarrow 0$. The first negative term in Eq. (24) is just related to the well-known Coulomb potential and the second and third positive terms come from the nonconformality appearance. The interesting point is that in high energy limit, $l \Lambda_{c} \ll 1$, by increasing $l \Lambda_{c}$ one can deduce that $\tilde{V}_{q \bar{q}}$ becomes less binding, i.e., the more nonconformality effects, the less binding dimensionless meson potential energy.

\section{B. Potential energy: finite temperature expansion}

In this section we would like to study the thermal physics of meson potential energy $V_{q \bar{q}}$ in the MBH background (2). We use the systematic expansion as we did in the previous section in both low temperature i.e., $l T \ll 1$ ( or $r_{h} \ll r_{*}$ ) and high temperature i.e., $l T \gg 1$ (or $r_{h} \sim r_{*}$ ) limits. Using (2), (16), and following the same previous calculations we reach the following expressions for $l$ and $V_{q \bar{q}}$

$$
\begin{aligned}
l\left(r_{*}\right)= & \frac{2}{r_{*}} \int_{0}^{1} \frac{\sqrt{f\left(r_{*}\right)}}{f(u)} u^{2} e^{\left(\frac{r_{c}}{r_{*}}\right)^{2}\left(1-u^{2}\right)} \\
& \times\left(1-\frac{f\left(r_{*}\right)}{f(u)} e^{2\left(\frac{r_{c}}{r_{*}}\right)^{2}\left(1-u^{2}\right)} u^{4}\right)^{-\frac{1}{2}} d u,
\end{aligned}
$$

$$
V_{q \bar{q}}(l)=\frac{r_{*}}{\pi \alpha^{\prime}} \int_{\delta}^{1} u^{-2} e^{\left(\frac{r_{c}}{r_{*}}\right)^{2} u^{2}}\left(1-\frac{f\left(r_{*}\right)}{f(u)} e^{2\left(\frac{r_{c}}{r_{*}}\right)^{2}\left(1-u^{2}\right)} u^{4}\right)^{-\frac{1}{2}} d u,
$$

where $\delta$ is an ultraviolet cutoff and $f(u)=1-\left(r_{h} / r_{*}\right)^{4} u^{4}$. The above integrals can not be solved analytically, so we develop a systematic expansion by using the binomial expansion. If we define $x \equiv\left(f\left(r_{*}\right) / f(u)\right) e^{2\left(\frac{r_{*}}{r_{*}}\right)^{2}\left(1-u^{2}\right)} u^{4}$, we will then get a convergent series, following the fact that $r_{c}<r_{*}$ and $r_{h}<r_{*}$. Now, (25) and (26) are given by

$$
\begin{aligned}
l\left(r_{*}\right)= & \frac{2}{r_{*}} \sum_{n=0}^{\infty} \frac{\Gamma\left(n+\frac{1}{2}\right)}{\sqrt{\pi} \Gamma(n+1)} f\left(r_{*}\right)^{n+\frac{1}{2}} \\
& \times \int_{0}^{1} \frac{u^{4 n+2}}{f(u)^{n+1}} e^{(2 n+1)\left(1-u^{2}\right)\left(\frac{r_{c}}{r_{*}}\right)^{2}} d u, \\
V_{q \bar{q}}\left(r_{*}\right)= & \frac{r_{*}}{\pi \alpha^{\prime}} \sum_{n=0}^{\infty} \frac{\Gamma\left(n+\frac{1}{2}\right)}{\sqrt{\pi} \Gamma(n+1)} f\left(r_{*}\right)^{n} \\
& \times \int_{\delta}^{1} \frac{u^{4 n-2}}{f(u)^{n}} e^{\left(2 n+(1-2 n) u^{2}\right)\left(\frac{r_{c}}{*_{*}}\right)^{2}} d u .
\end{aligned}
$$

Again, in order to find $V_{q \bar{q}}$ in terms of $l$ we should solve Eq. (27) for $r_{*}$ and use it in (28) to obtain $V_{q \bar{q}}$ as a function of $l$. Practically, it is not possible to solve Eq. (27) analytically to find $r_{*}$ as a function of $l$. However, we can study the behavior of meson potential energy in low and high temperature limits, in the high energy limit defined as $l \Lambda_{c} \ll 1$ (or $r_{c} \ll r_{*}$ ).

\section{Low temperature limit}

At low temperature $(l T \ll 1)$, the extremized classical string world sheet is positioned next to the boundary and thus the leading contribution comes from the near boundary expansion. Finite temperature corrections and nonconformal effects appear as subleading terms corresponding to the deviation of the bulk geometry from pure AdS. Focusing on the high energy limit, and using Eq. (27), we reach the following expression for $l$ up to the fourth order in $r_{c} / r_{*}$ and $r_{h} / r_{*}$

$$
\begin{aligned}
l\left(r_{*}\right)= & \frac{2}{r_{*}}\left[a_{1}+\alpha_{1}\left(\frac{r_{h}}{r_{*}}\right)^{4}+\left(a_{2}+\alpha_{2}\left(\frac{r_{h}}{r_{*}}\right)^{4}\right)\left(\frac{r_{c}}{r_{*}}\right)^{2}\right. \\
& \left.+\left(a_{3}+\alpha_{3}\left(\frac{r_{h}}{r_{*}}\right)^{4}\right)\left(\frac{r_{c}}{r_{*}}\right)^{4}\right], \quad \alpha_{1}, \alpha_{2}, \alpha_{3}<0,
\end{aligned}
$$

where $\alpha_{1}, \alpha_{2}$ and $\alpha_{3}$ are numerical coefficients reported in Table II in Appendix A. Solving Eq. (29) perturbatively for $r_{*}$ we obtain a perturbative expression for meson potential energy by considering the finite parts (the details of the calculations are written in Appendix A) 


$$
\begin{aligned}
\hat{V}_{q \bar{q}}\left(l \Lambda_{c}, l T\right) \equiv & \frac{\alpha^{\prime} V_{q \bar{q}}\left(l, l \Lambda_{c}, l T\right)}{\Lambda_{c}^{\frac{1}{2}} T^{\frac{1}{2}}} \\
= & \frac{1}{l \Lambda_{c}^{\frac{1}{2}} T^{\frac{1}{2}}}\left[\bar{b}_{1}+\bar{\beta}_{1}(l T)^{4}+\frac{1}{2}\left(\bar{b}_{2}+\bar{\beta}_{2}(l T)^{4}\right)\left(l \Lambda_{c}\right)^{2}\right. \\
& \left.+\frac{1}{4}\left(\bar{b}_{3}+\bar{\beta}_{3}(l T)^{4}\right)\left(l \Lambda_{c}\right)^{4}\right] \\
& \bar{\beta}_{1}<0, \bar{\beta}_{2}, \bar{\beta}_{3}>0
\end{aligned}
$$

where $\hat{V}_{q \bar{q}}$ is the dimensionless meson potential energy at low temperature and $\bar{\beta}_{1}, \bar{\beta}_{2}$ and $\bar{\beta}_{3}$ are numerical coefficients given in Appendix A and reported in Table II. Note that we make the meson potential energy dimensionless in such a way that we can get the intuitive results by taking the limits $l \Lambda_{c} \rightarrow 0$ and/or $l T \rightarrow 0$ of (30). The first two terms indicate the known meson potential energy for pure AdS and AdS black hole, respectively, and the next two terms are thermal and nonconformal corrections. In the high energy and low temperature limit, if we fix $l T$ and increase $l \Lambda_{c}$ we will then find that $\hat{V}_{q \bar{q}}$ becomes less binding and the same results will be achieved by fixing $l \Lambda_{c}$ and increasing $l T$. We summarize the results corresponding to the mentioned limits:

(a) $l \Lambda_{c} \rightarrow 0, l T$ finite and small enough: We get the zero temperature meson potential energy as the leading term and the subleading term, the term including $\bar{\beta}_{1}<0$, is the finite temperature correction which makes the meson potential energy less binding.

(b) $l T \rightarrow 0, l \Lambda_{c}$ finite and small enough: The leading term is the conformal meson potential energy and the nonconformal terms, including $\bar{b}_{2}>0$ and $\bar{b}_{3}>0$ terms, appear as the subleading terms. Clearly, these corrections cause the meson potential energy to be less binding.

(c) $l \Lambda_{c} \rightarrow 0$ and $l T \rightarrow 0$ : Obviously, we face the conformal meson potential energy.

According to the gauge/gravity dictionary, there is a phase transition point where $r_{h} \rightarrow r_{c}$ [35] which is equivalent to the limit $T \rightarrow \Lambda_{c} / \pi \sqrt{2}$ and we call it the transition limit. We would like to study the meson potential energy near this point, hence we take the transition limit of $\hat{V}_{q \bar{q}}$. Doing some algebra, up to fourth order in $l \Lambda_{c}$ we reach the following expression

$$
\begin{aligned}
& \left.\hat{V}_{q \bar{q}}\left(l \Lambda_{c}, l T\right)\right|_{T \rightarrow \frac{\Lambda_{c}}{\pi \sqrt{2}}} \\
& \quad=\frac{1}{l \Lambda_{c}}\left[\bar{b}_{1}+\frac{\bar{b}_{2}}{2}\left(l \Lambda_{c}\right)^{2}+\left(\frac{\bar{b}_{3}}{4}+\frac{\bar{\beta}_{1}}{4 \pi^{4}}\right)\left(l \Lambda_{c}\right)^{4}\right] .
\end{aligned}
$$

To get a better understanding of the meson potential energy near transition point, one can fix $l \Lambda_{c}$ and compare the zero temperature potential energy $\tilde{V}_{q \bar{q}}$ with the finite temperature one $\hat{V}_{q \bar{q}}$ in the transition limit. To do so, we should focus on the difference between Eqs. (24) and (31)

$\left|\hat{V}_{q \bar{q}}\left(l \Lambda_{c}, l T\right)\right|_{T \rightarrow \frac{\Lambda_{c}}{\pi \sqrt{2}}}-\left|\tilde{V}_{q \bar{q}}\left(l \Lambda_{c}\right)\right|=\frac{\left|\bar{\beta}_{1}\right|}{4 \pi^{4}}\left(l \Lambda_{c}\right)^{3}>0$.

Near the phase transition point, it is seen that the zero temperature dimensionless meson potential energy is less binding; that is the amount of energy required to break down the meson to quark and antiquark is less. We will return to this issue later in the paper.

\section{High temperature limit}

At high temperatures, i.e., $l T \gg 1$ ( or $r_{*} \sim r_{h}$ ), the extremized classical string world sheet tends to reach the horizon and therefore the leading contribution is related to the near horizon background. Since we work in the high energy limit, $r_{c} \ll r_{*}$, the high temperature limit can be identified by $r_{h} \rightarrow r_{*}$. As $r_{*}$ touches the horizon the classical string minimizes its energy by splitting into two vertical strings ending at the horizon. At high temperatures, $r_{h}$ approaches $r_{*}$ and hence $f\left(r_{*}\right)=1-r_{h}^{4} / r_{*}^{4} \rightarrow 0$, one can see from (28) that the meson potential energy is zero and there is no more information regarding the high temperature expansion of the meson potential energy.

\section{HOLOGRAPHIC SUBREGION COMPLEXITY}

In this section we would like to study HSC using the volume prescription written in (12) for the MAdS and MBH backgrounds [Eqs. (1) and (2), respectively].

\section{A. Holographic subregion complexity: zero temperature expansion}

We consider a striplike boundary region $A$ in the $\vec{x}$ direction at a constant time slice. This region can be parametrized as

$-\frac{l}{2} \leq x \equiv x(r) \leq \frac{l}{2}, \quad-\frac{L}{2} \leq y, z \leq \frac{L}{2}, \quad L \gg l$.

Extremal surface is translationally invariant along $y$ and $z$ axes and the profile of the surface on the bulk is $x(r)$. If one identifies the quark/antiquark separation, characteristic length $l$, with subregion length, HSC of $A$ can be considered as the complexity of the probe meson in nonconformal vacuum. According to (12), we need to obtain the volume enclosed by subregion $A$ and RT surface which we call $V_{\gamma_{A}}$. At zero temperature using the MAdS background (1) we get

$$
V_{\gamma_{A}}=2 L^{2} \int_{r_{*}}^{\infty} r^{2} e^{\frac{c}{r^{2}}} x(r) d r
$$

where $L^{2}$ is the volume of corresponding $y z$ plane. To find $x(r)$ we need to compute the area of the RT surface 


$$
A=2 L^{2} \int_{0}^{\frac{l}{2}} r^{2} e^{\frac{3 c}{4 r^{2}}} \sqrt{r^{2}+\frac{r^{\prime 2}}{r^{2}}} d x
$$

Solving the equation of motion for $x(r)$, the expressions for $V_{\gamma_{A}}$ and $l$ are given by

$$
\begin{aligned}
l\left(r_{*}\right)= & \frac{2}{r_{*}} \int_{0}^{1} u^{3} e^{\frac{3}{2}\left(\frac{r_{c}}{r_{*}}\right)^{2}\left(1-u^{2}\right)}\left(1-e^{3\left(\frac{r c}{r_{*}}\right)^{2}\left(1-u^{2}\right)} u^{6}\right)^{-\frac{1}{2}} d u, \\
V_{\gamma_{A}}\left(r_{*}\right)= & 2 L^{2} r_{*}^{2} \int_{\delta}^{1} u^{-4} e^{2\left(\frac{r r c}{r_{*}}\right)^{2} u^{2}} \\
& \times \int_{u}^{1} u^{3} e^{\frac{3}{2}\left(\frac{r_{c}}{r_{*}}\right)^{2}\left(1-u^{2}\right)}\left(1-e^{3\left(\frac{r c}{r_{*}}\right)^{2}\left(1-u^{2}\right)} u^{6}\right)^{-\frac{1}{2}} d u,
\end{aligned}
$$

where again $u=r_{*} / r$ and $\delta$ is an ultraviolet cutoff introduced since the volume integral is divergent. This integration is not solvable analytically. Hence, we use the same method, systematic expansion, in Sec. IV A and then (36) and (37) can be written as follows:

$l\left(r_{*}\right)=\frac{2}{r_{*}} \sum_{n=0}^{\infty} \frac{\Gamma\left(n+\frac{1}{2}\right)}{\sqrt{\pi} \Gamma(n+1)} \int_{0}^{1} u^{6 n+3} e^{\frac{3}{2}(2 n+1)\left(1-u^{2}\right)\left(\frac{r_{*}}{r_{*}}\right)^{2}} d u$,

$$
\begin{aligned}
V_{\gamma_{A}}\left(r_{*}\right)= & 2 L^{2} r_{*}^{2} \sum_{n=0}^{\infty} \frac{\Gamma\left(n+\frac{1}{2}\right)}{\sqrt{\pi} \Gamma(n+1)} \int_{\delta}^{1} u^{-4} e^{2\left(\frac{r c}{r_{*}}\right)^{2} u^{2}} \\
& \times \int_{u}^{1} u^{6 n+3} e^{\frac{3}{2}(2 n+1)\left(1-u^{2}\right)\left(\frac{r_{c}}{r_{*}}\right)^{2}} d u .
\end{aligned}
$$

Again to find HSC as a function of $l$ we need to solve Eq. (38) for $r_{*}$ and then put it in Eq. (39) to obtain $V_{\gamma_{A}}$ in terms of $l$. HSC can be computed using Eq. (12). In practice, this procedure can not be performed analytically and hence we focus on high energy limit, $r_{c} \ll r_{*}$.

\section{High energy limit}

In the high energy limit, from Eq. (38) and keeping up to the fourth order in $r_{c} / r_{*}$ we get

$l\left(r_{*}\right)=\frac{2}{r_{*}}\left[k_{1}+k_{2}\left(\frac{r_{c}}{r_{*}}\right)^{2}+k_{3}\left(\frac{r_{c}}{r_{*}}\right)^{4}\right], k_{1}, k_{2}, k_{3}>0$

where $k_{1}, k_{2}$ and $k_{3}$ are numerical coefficients reported in Table III in Appendix B. If we solve Eq. (40) perturbatively for $r_{*}$ then we will finally obtain the following finite expression (for more details of the calculation refer to Appendix B)

$$
\begin{aligned}
\tilde{V}_{\gamma_{A}}\left(l \Lambda_{c}\right) \equiv & \frac{V_{\gamma_{A}}\left(l, l \Lambda_{c}\right)}{L^{2} \Lambda_{c}^{2}} \\
= & \frac{1}{\left(l \Lambda_{c}\right)^{2}}\left(\bar{w}_{1}+\frac{\bar{w}_{2}}{2}\left(l \Lambda_{c}\right)^{2}+\frac{\bar{w}_{3}}{4}\left(l \Lambda_{c}\right)^{4}\right), \\
& \bar{w}_{1}, \bar{w}_{2}, \bar{w}_{3}<0,
\end{aligned}
$$

where $\tilde{V}_{\gamma_{A}}$ is dimensionless volume and $\bar{w}_{1}, \bar{w}_{2}$ and $\bar{w}_{3}$ are numerical coefficients shown in Table III in Appendix B. Finally, HSC can be obtained using Eqs. (12) and (41). The first negative term contributes to the boundary of MAdS. The second and third negative terms correspond to the nonconformality effects. In the high energy limit, it is seen that increasing $l \Lambda_{c}$ causes $\tilde{V}_{\gamma_{A}}$ to decrease. In other words, the more nonconformality effects, the less required information to specify the meson state. On the other hand, as we mentioned in Sec. IVA 1, the more nonconformality effects make less binding dimensionless meson potential energy. In order to get a better understanding of a relation between HSC and meson potential energy, we fix $l \Lambda_{c}$ and then the nicely linear relation between $\tilde{V}_{q \bar{q}}$ and $\tilde{V}_{\gamma_{A}}$ using (24) and (41) is given by

$$
\frac{\tilde{V}_{\gamma_{A}}\left(l \Lambda_{c}\right)}{\tilde{V}_{q \bar{q}}\left(l \Lambda_{c}\right)}=a_{0}\left(l \Lambda_{c}\right),
$$

where, at leading order, $a_{0}$ is a decreasing function of $l \Lambda_{c}$ in the high energy limit. Due to the above linear relation one can deduce that the less binding meson state needs less information to be specified and vice versa. In Fig. 1, we plot the relation (42) for two different values of $l \Lambda_{c}$. It is seen that, if one considers a meson state with specific $\tilde{V}_{q \bar{q}}$ and increases (decreases) $l \Lambda_{c}$, then less (more) information will be needed to obtain the mentioned state. However, it seems that the nonconformality effect does not respect this result, i.e., in nonconformal field theory a less binding meson needs more information to specify.

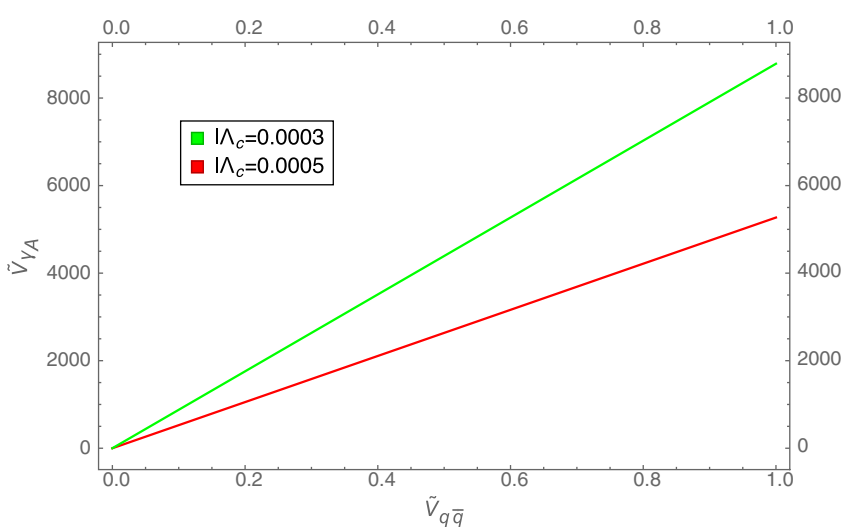

FIG. 1. At leading order, the dimensionless volume $\tilde{V}_{\gamma_{A}}$ in terms of the dimensionless meson potential energy $\tilde{V}_{q \bar{q}}$ for fixed values of $l \Lambda_{c}$ at zero temperature. The different curves correspond to different values of $l \Lambda_{c}=0.0003$ (green curve) and $l \Lambda_{c}=0.0005$ (red curve). 


\section{B. Holographic subregion complexity: finite temperature expansion}

Here, we investigate the thermal behavior of HSC in the MBH background (2). We develop a systematic expansion in low temperature $l T \ll 1$ ( or $r_{h} \ll r_{*}$ ) and high temperature $l T \gg 1$ ( or $r_{*} \sim r_{h}$ ) limits and focus on the high energy limit $l \Lambda_{c} \ll 1$ (or $\left.r_{c} \ll r_{*}\right)$. The same calculations lead to the following expressions for $l$ and $V_{\gamma_{A}}$,

$l\left(r_{*}\right)=\frac{2}{r_{*}} \int_{0}^{1} \frac{u^{3}}{\sqrt{f(u)}} e^{\frac{3}{2}\left(\frac{r_{c}}{r_{*}}\right)^{2}\left(1-u^{2}\right)}\left(1-e^{3\left(\frac{r c}{r_{*}}\right)^{2}\left(1-u^{2}\right)} u^{6}\right)^{-\frac{1}{2}} d u$,

$$
\begin{aligned}
V_{\gamma_{A}}\left(r_{*}\right)= & 2 L^{2} r_{*}^{2} \int_{\delta}^{1} \frac{u^{-4}}{\sqrt{f(u)}} e^{2\left(\frac{r c}{r_{*}}\right)^{2} u^{2}} \\
& \times \int_{u}^{1} \frac{u^{3}}{\sqrt{f(u)}} e^{\frac{3}{2}\left(\frac{r_{*}}{r_{*}}\right)^{2}\left(1-u^{2}\right)}\left(1-e^{3\left(\frac{r_{c}}{r_{*}}\right)^{2}\left(1-u^{2}\right)} u^{6}\right)^{-\frac{1}{2}} d u,
\end{aligned}
$$

where $\delta$ is an ultraviolet cutoff and $f(u)=1-\left(r_{h} / r_{*}\right)^{4} u^{4}$. Again, analogous to the Sec. IV B, the above integrals can not be solved analytically and hence we can develop a systematic expansion. Using binomial expansions equations (43) and (44) are given by

$$
\begin{aligned}
l\left(r_{*}\right) & =\frac{2}{r_{*}} \sum_{n=0}^{\infty} \sum_{m=0}^{\infty} \frac{\Gamma\left(n+\frac{1}{2}\right) \Gamma\left(m+\frac{1}{2}\right)}{\pi \Gamma(n+1) \Gamma(m+1)} \int_{0}^{1}\left(\frac{r_{h}}{r_{*}}\right)^{4 m} u^{4 m+6 n+3} e^{\frac{3}{2}(2 n+1)\left(1-u^{2}\right)\left(\frac{r_{c}}{r_{*}}\right)^{2}} d u, \\
V_{\gamma_{A}}\left(r_{*}\right)= & 2 V_{y, z} r_{*}^{2} \sum_{n=0}^{\infty} \sum_{m=0}^{\infty} \sum_{p=0}^{\infty} \frac{\Gamma\left(n+\frac{1}{2}\right) \Gamma\left(m+\frac{1}{2}\right) \Gamma\left(p+\frac{1}{2}\right)}{\pi^{3 / 2} \Gamma(n+1) \Gamma(m+1) \Gamma(p+1)} \int_{\delta}^{1}\left(\frac{r_{h}}{r_{*}}\right)^{4 p} u^{4 p-4} e^{2\left(\frac{r_{*}}{r_{*}}\right)^{2} u^{2}} \\
& \times \int_{u}^{1}\left(\frac{r_{h}}{r_{*}}\right)^{4 m} u^{4 m+6 n+3} e^{\frac{3}{2}(2 n+1)\left(1-u^{2}\right)\left(\frac{r_{c}}{r_{*}}\right)^{2}} d u
\end{aligned}
$$

Since $r_{c}<r_{*}$ and $r_{h}<r_{*}$ we do not worry about the convergence of the above series and hence these are well defined. Now, the rest of the procedure is familiar. We have to take the following steps: Solving Eq. (45) for $r_{*}$ and then calculating $V_{\gamma_{A}}$ by using Eq. (46) and finally computing HSC using Eq. (12). Unfortunately, this process cannot be done analytically and hence we have to study the behavior of the HSC at low and high temperatures, in the high energy limit.

\section{Low temperature limit}

At low temperature $l T \ll 1$, the extremal surface is restricted to be near the boundary and hence the leading contribution to the HSC comes from near AdS boundary expansion. Therefore, we should expect the zero temperature HSC to be the leading term. Finite temperature corrections correspond to the deviation of the bulk geometry from pure AdS. Working on the high energy limit, and using (45), we get the following expression for $l$ up to the fourth order in $r_{c} / r_{*}$ and $r_{h} / r_{*}$ we have

$$
\begin{aligned}
l\left(r_{*}\right)= & \frac{2}{r_{*}}\left[k_{1}+\kappa_{1}\left(\frac{r_{h}}{r_{*}}\right)^{4}+\left(k_{2}+\kappa_{2}\left(\frac{r_{h}}{r_{*}}\right)^{4}\right)\left(\frac{r_{c}}{r_{*}}\right)^{2}\right. \\
& \left.+\left(k_{3}+\kappa_{3}\left(\frac{r_{h}}{r_{*}}\right)^{4}\right)\left(\frac{r_{c}}{r_{*}}\right)^{4}\right], \quad \kappa_{1}, \kappa_{2}, \kappa_{3}>0
\end{aligned}
$$

where $\kappa_{1}, \kappa_{2}, \kappa_{3}$ are numerical coefficients reported in Table IV in Appendix B. Solving Eq. (47) perturbatively for $r_{*}$ we reach a perturbative finite expression

$$
\begin{aligned}
\hat{V}_{\gamma_{A}}\left(l \Lambda_{c}, l T\right) \equiv & \frac{V_{\gamma_{A}}\left(l, l \Lambda_{c}, l T\right)}{L^{2} \Lambda_{c} T} \\
= & \frac{1}{l^{2} \Lambda_{c} T}\left(\bar{w}_{1}+\bar{\omega}_{1}(l T)^{4}\right. \\
& +\frac{1}{2}\left(\bar{w}_{2}+\bar{\omega}_{2}(l T)^{4}\right)\left(l \Lambda_{c}\right)^{2} \\
& \left.+\frac{1}{4}\left(\bar{w}_{3}+\bar{\omega}_{3}(l T)^{4}\right)\left(l \Lambda_{c}\right)^{4}\right), \\
& \bar{\omega}_{1}, \bar{\omega}_{2}<0, \quad \bar{\omega}_{3}>0,
\end{aligned}
$$

where $\hat{V}_{\gamma_{A}}$ is dimensionless volume at low temperature and $\bar{\omega}_{1}, \bar{\omega}_{2}$ and $\bar{\omega}_{3}$ are numerical coefficients reported in Table IV in Appendix B (the details of the calculation are in Appendix B). HSC can be computed using the relation (12). We make $V_{\gamma_{A}}$ dimensionless to have meaningful and intuitive limits which are favorable such as $l \Lambda_{c} \rightarrow 0$ and/or $l T \rightarrow 0$. The first two terms are the known results corresponding to the pure AdS and AdS black hole HSC, respectively, and the next two terms indicate the thermal and nonconformal corrections. From Eq. (48), it is seen that if one fixes $l T$ and increases $l \Lambda_{c}$, then $\hat{V}_{\gamma_{A}}$ will decrease and the same results will be obtained by changing 
the role of $l T$ and $l \Lambda_{c}$. This result can be clearly observed from Fig. 2 where we plot $\hat{V}_{\gamma_{A}}$ as a function of $l T\left(l \Lambda_{c}\right)$ for fixed value of $l \Lambda_{c}(l T)$. Interestingly, following the results we get for $\hat{V}_{q \bar{q}}$ in the Sec. IV B 1, one can conclude that the less binding $\hat{V}_{q \bar{q}}$ the less information required to specify the meson state, at leading order, and the thermal effects may not respect to this result. Below, we represent the results corresponding to the desired limits of Eq. (48).

(a) $l \Lambda_{c} \rightarrow 0, l T$ finite and small enough: The leading term corresponding to zero temperature HSC and the subleading term, the term including $\bar{\omega}_{1}<0$, is the finite temperature correction which leads to a decline in corresponding HSC.

(b) $l T \rightarrow 0, l \Lambda_{c}$ finite and small enough: The leading term corresponding to the conformal HSC and the nonconformal corrections, including $\bar{\omega}_{2}<0$ and $\bar{\omega}_{3}<0$ terms, appear as the subleading terms. These corrections lead to a decrease in corresponding HSC.

(c) $l \Lambda_{c} \rightarrow 0$ and $l T \rightarrow 0$ : Clearly, we reach the corresponding conformal HSC.

We would like to study HSC near the transition point, by taking the transition limit $T \rightarrow \Lambda_{c} / \pi \sqrt{2}$ of Eq. (48). Up to the fourth order in $l \Lambda_{c}$ we get

$$
\begin{aligned}
\hat{V}_{\gamma_{A}} & =\left.\left(l \Lambda_{c}, l T\right)\right|_{T \rightarrow \frac{\Lambda_{c}}{\pi \sqrt{2}}} \\
& =\frac{1}{\left(l \Lambda_{c}\right)^{2}}\left[\bar{w}_{1}+\frac{\bar{w}_{2}}{2}\left(l \Lambda_{c}\right)^{2}+\left(\frac{\bar{w}_{3}}{4}+\frac{\bar{\omega}_{1}}{4 \pi^{4}}\right)\left(l \Lambda_{c}\right)^{4}\right] .
\end{aligned}
$$

We fix $l \Lambda_{c}$ and compare the HSC at zero temperature (41) and finite temperature (49) in the transition limit. We have

$$
\left|\hat{V}_{\gamma_{A}}\left(l \Lambda_{c}, l T\right)\right|_{T \rightarrow \frac{\Lambda_{c}}{\pi \sqrt{2}}}-\left|\tilde{V}_{\gamma_{A}}\left(l \Lambda_{c}\right)\right|=\frac{\left|\bar{\omega}_{1}\right|}{4 \pi^{4}}\left(l \Lambda_{c}\right)^{3}>0 .
$$

If we accept that a state which needs less information to be specified is a favorable state, then Eq. (50) will indicate that near the phase transition point the meson state at zero temperature is favorable. Additionally, according to Eq. (32) the zero temperature meson state is less binding. Therefore, in short, near the transition point the less binding meson is more favorable, i.e., it needs less information to specify in the mentioned limits. Notice that by taking the transition limit, the meson state at finite temperature is more binding. These results are in agreement with [26].

We would like to obtain a relation between HSC and meson potential energy at low temperature. If we fix $l \Lambda_{c}$ and $l T$, then using (30) and (48) we will reach a linear relation between $\hat{V}_{q \bar{q}}$ and $\hat{V}_{\gamma_{A}}$ at low temperature and leading order, the same as the zero temperature case, which is given by

$$
\frac{\hat{V}_{\gamma_{A}}\left(l \Lambda_{c}, l T\right)}{\hat{V}_{q \bar{q}}\left(l \Lambda_{c}, l T\right)}=a_{T}\left(l \Lambda_{c}, l T\right),
$$

where $a_{T}$ is a decreasing function of $l \Lambda_{c}$ and $l T$ at low temperature and high energy. According to (51) the less binding meson state needs less information to be specified and vice versa. In Fig. 3, we plot relation (51) for different values of $l \Lambda_{c}$ and $l T$. In the left, we fix $l T$ and consider different values of $l \Lambda_{c}$ and in the right we fix $l \Lambda_{c}$ and consider different values of $l T$. From the left figure, it is seen that if one considers a meson state with specific $\hat{V}_{q \bar{q}}$, then by increasing (decreasing) $l \Lambda_{c}$ at fixed $l T$ the less (more) information will be needed to determine that state. On the other hand, the right figure shows by increasing (decreasing) $l T$ at fixed $l \Lambda_{c}$ the less (more) information will be needed to specify that state.

\section{High temperature limit}

At high temperature, i.e., $l T \gg 1$ (or $r_{*} \sim r_{h}$ ), the thermal fluctuations become considerable and the extremal

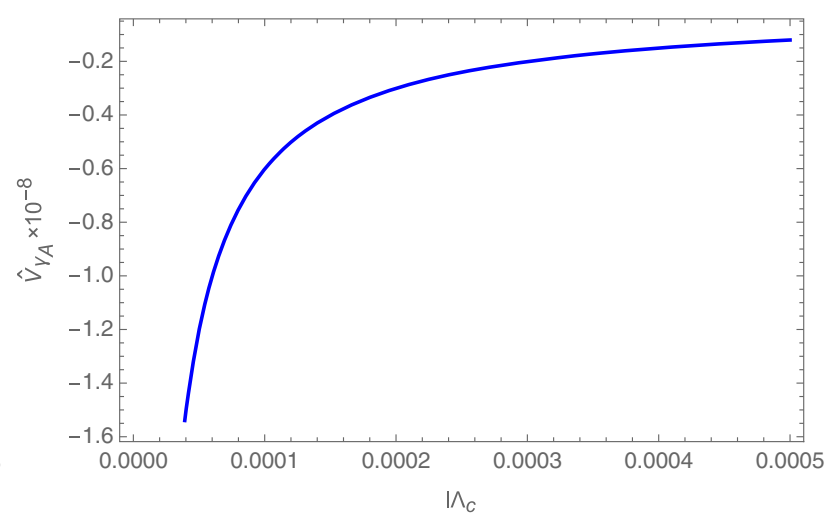

FIG. 2. Left: At leading order, the dimensionless volume $\hat{V}_{\gamma_{A}}$ as a function of $l T$ for fixed $l \Lambda_{c}=0.0001$, in the low temperature limit. Right: The dimensionless subregion volume $\hat{V}_{\gamma_{A}}$ as a function of $l \Lambda_{c}$ for fixed $l T=0.0001$, in the low temperature limit. 

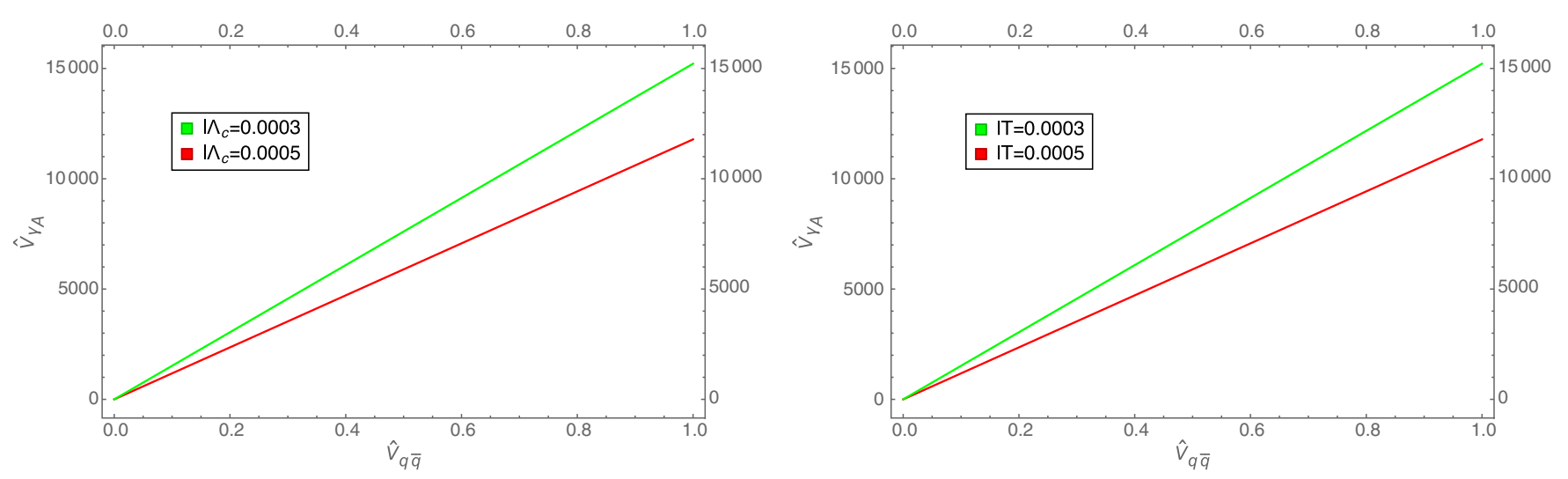

FIG. 3. Left: At leading order, the dimensionless volume $\hat{V}_{\gamma_{A}}$ as a function of the dimensionless meson potential energy $\hat{V}_{q \bar{q}}$ for fixed values of $l \Lambda_{c}$ in the low temperature limit. The different curves correspond to different values of $l \Lambda_{c}=0.0003$ (green curve) and $l \Lambda_{c}=0.0005$ (red curve). we fix $l T=0.0001$. Right: $\hat{V}_{\gamma_{A}}$ as a function of $\hat{V}_{q \bar{q}}$ for fixed values of $l T, l T=0.0003$ (green curve) and $l T=0.0005$ (red curve) in the low temperature limit. We fix $l \Lambda_{c}=0.0001$.

surface gets close to the horizon. The leading contribution comes from the near horizon background and the full bulk contributes to the subleading terms. In high temperature and high energy limit $r_{c} \ll r_{*}$ and $r_{h} \rightarrow r_{*}$, respectively, we integrate Eqs. (45) and (46) and consider finite parts, up to $\left(r_{c} / r_{*}\right)^{2}$ and we get

$$
\begin{gathered}
l\left(r_{*}\right)=\frac{2}{r_{*}} \sum_{n=0}^{\infty} \sum_{m=0}^{\infty} \frac{\Gamma\left(n+\frac{1}{2}\right) \Gamma\left(m+\frac{1}{2}\right)}{\pi \Gamma(n+1) \Gamma(m+1)}\left(\frac{r_{h}}{r_{*}}\right)^{4 m}\left[L_{1}+L_{2}\left(\frac{r_{c}}{r_{*}}\right)^{2}\right], \\
V_{\gamma_{A}}=2 L^{2} r_{*}^{2} \sum_{n=0}^{\infty} \sum_{m=0}^{\infty} \sum_{p=0}^{\infty} \frac{\Gamma\left(n+\frac{1}{2}\right) \Gamma\left(m+\frac{1}{2}\right) \Gamma\left(p+\frac{1}{2}\right)}{\pi^{3 / 2} \Gamma(n+1) \Gamma(m+1) \Gamma(p+1)}\left(\frac{r_{h}}{r_{*}}\right)^{4(m+p)}\left[C_{1}+C_{2}\left(\frac{r_{c}}{r_{*}}\right)^{2}\right],
\end{gathered}
$$

where $L_{1}, L_{2}, C_{1}$ and $C_{2}$ are constant coefficients given by

$$
\begin{aligned}
L_{1}= & \frac{1}{6 n+4 m+4}, \\
L_{2}= & \frac{3}{2}(2 n+1)\left(\frac{1}{6 n+4 m+4}-\frac{1}{6 n+4 m+6}\right), \\
C_{1}= & \frac{1}{(6 n+4 m+4)(4 p-3)}-\frac{1}{(6 n+4 m+4)(6 n+4 m+4 p+1)}, \\
C_{2}= & \frac{3}{2}(2 n+1)\left(\frac{1}{(6 n+4 m+4)(4 p-3)}-\frac{1}{(6 n+4 m+4)(6 n+4 m+4 p+1)}\right. \\
& \left.-\frac{1}{(6 n+4 m+6)(4 p-3)}+\frac{1}{(6 n+4 m+6)(6 n+4 m+4 p+3)}\right) \\
& +\frac{2}{(6 n+4 m+4)(4 p-1)}-\frac{2}{(6 n+4 m+4)(6 n+4 m+4 p+3)} .
\end{aligned}
$$

The next step is to write $V_{\gamma_{A}}$ in terms of $l$. To do so, we perform the calculations order by order up to $\left(r_{c} / r_{*}\right)^{2}$ and check carefully the convergence of the resulting infinite series. 
(a) Up to $\left(r_{c} / r_{*}\right)^{0}$ : We consider the terms including $L_{1}$ and $C_{1}$ which are the known results for AdS black hole. Using Eqs. (52) and (53), we obtain

$$
\frac{V_{\gamma_{A}}}{L^{2}}=l r_{*}^{3}\left(\sum_{p=1}^{\infty} \frac{\Gamma\left(p+\frac{1}{2}\right)}{\sqrt{\pi} \Gamma(p+1)(4 p-3)}\left(\frac{r_{h}}{r_{*}}\right)^{4 p}-\frac{1}{3}\right)-2 r_{*}^{2} \mathcal{A}
$$

where the term $-1 / 3$ corresponds to $p=0$ term and expresses the fact that only $p=0$ term is divergent and $\mathcal{A}$ is given by

$$
\mathcal{A}=\sum_{n=0}^{\infty} \sum_{m=0}^{\infty} \sum_{p=0}^{\infty} \frac{\Gamma\left(n+\frac{1}{2}\right) \Gamma\left(m+\frac{1}{2}\right) \Gamma\left(p+\frac{1}{2}\right)}{\pi^{3 / 2} \Gamma(n+1) \Gamma(m+1) \Gamma(p+1)(6 n+4 m+4)(6 n+4 m+4 p+1)}\left(\frac{r_{h}}{r_{*}}\right)^{4(m+p)} .
$$

For large $p$, the first infinite series in (55) goes as $\sim p^{-3 / 2}\left(r_{h} / r_{*}\right)^{4 p}$ and hence converges for $r_{h}=r_{*}$. We are in a position to check the convergence of (56), then we sum over $n$ and $p$ in (56) and obtain

$$
\begin{aligned}
\mathcal{A}= & \sum_{m=0}^{\infty}\left(\frac{r_{h}}{r_{*}}\right)^{4 m} \frac{\Gamma\left(m+\frac{1}{2}\right)}{720 \Gamma(m+1)}\left[-\frac{40 \Gamma\left(\frac{2 m}{3}+\frac{2}{3}\right)}{\Gamma\left(\frac{2 m}{3}+\frac{7}{6}\right)}{ }_{2} F_{1}\left(-\frac{3}{4}, \frac{1}{2} ; \frac{1}{4} ;\left(\frac{r_{h}}{r_{*}}\right)^{4}\right)\right. \\
& +\frac{40 \Gamma\left(\frac{2 m}{3}+\frac{1}{6}\right)}{\Gamma\left(\frac{2 m}{3}+\frac{2}{3}\right)}{ }_{6} F_{5}\left(-\frac{1}{4}, \frac{1}{6}, \frac{1}{2}, \frac{5}{6}, \frac{m}{3}+\frac{1}{12}, \frac{m}{3}+\frac{7}{12} ; \frac{1}{3}, \frac{2}{3}, \frac{3}{4}, \frac{m}{3}+\frac{1}{3}, \frac{m}{3}+\frac{5}{6} ;\left(\frac{r_{h}}{r_{*}}\right)^{12}\right) \\
& -\frac{60 \Gamma\left(\frac{2 m}{3}+\frac{5}{6}\right)}{\Gamma\left(\frac{2 m}{3}+\frac{4}{3}\right)}\left(\frac{r_{h}}{r_{*}}\right)^{4}{ }_{6} F_{5}\left(\frac{1}{12}, \frac{1}{2}, \frac{5}{6}, \frac{7}{6}, \frac{m}{3}+\frac{5}{12}, \frac{m}{3}+\frac{11}{12} ; \frac{2}{3}, \frac{13}{12}, \frac{4}{3}, \frac{m}{3}+\frac{2}{3}, \frac{m}{3}+\frac{7}{6} ;\left(\frac{r_{h}}{r_{*}}\right)^{12}\right) \\
& \left.-\frac{9 \Gamma\left(\frac{2 m}{3}+\frac{3}{2}\right)}{\Gamma\left(\frac{2 m}{3}+2\right)}\left(\frac{r_{h}}{r_{*}}\right)^{8}{ }_{6} F_{5}\left(\frac{5}{12}, \frac{5}{6}, \frac{7}{6}, \frac{3}{2}, \frac{m}{3}+\frac{3}{4}, \frac{m}{3}+\frac{5}{4} ; \frac{4}{3}, \frac{17}{12}, \frac{5}{3}, \frac{m}{3}+1, \frac{m}{3}+\frac{3}{2} ;\left(\frac{r_{h}}{r_{*}}\right)^{12}\right)\right],
\end{aligned}
$$

where ${ }_{p} F_{q}$ is the hypergeometric function and we use the following relation for $q=p-1$

${ }_{p} F_{q}\left(a_{1}, \ldots, a_{p} ; b_{1}, \ldots, b_{q} ; z\right)=\sum_{k=0}^{\infty} \frac{z^{k}}{k !} \frac{\left(a_{1}\right)_{k} \ldots\left(a_{p}\right)_{k}}{\left(b_{1}\right)_{k} \ldots\left(b_{q}\right)_{k}},|z|<1 \quad$ or $\quad|z|=1 \quad$ and $\operatorname{Re}\left(\sum_{j=1}^{q} b_{j}-\sum_{j=1}^{p} a_{j}\right)>0$,

where $(a)_{k} \equiv \Gamma(a+k) / \Gamma(a)$ is the Pochhamer symbol. Using Eqs. (58) it can be shown that for large $m$, each term of the sum (57) behaves as $\sim m^{-1}\left(r_{h} / r_{*}\right)^{4 m}$ and hence the series diverges for $r_{*}=r_{h}$. We should add and subtract the divergence piece of this series and then write $r_{*}=r_{h}(1+\epsilon)$ where at high temperature $\epsilon \ll 1$ and do an expansion for small $\epsilon$. We obtain

$$
\mathcal{A}=A_{1}+A_{2} \ln (4 \epsilon)+\mathcal{O}(\epsilon)
$$

where $A_{1}$ and $A_{2}$ are constant coefficients given in Appendix B and $\ln (4 \epsilon)$ is a function of $r_{h}$ and $l$ obtained in Appendix B. Using (55) and (59), we reach the following linear relation

$$
V_{H T}^{(0)}(l T) \equiv \frac{V_{\gamma_{A}}}{L^{2} T^{2}}=M_{1}^{(0)}+l T M_{2}^{(0)}
$$

where $V_{H T}^{(0)}$ is dimensionless volume up to the $\left(r_{c} / r_{*}\right)^{0}$ at high temperature, $M_{1}^{(0)}$ and $M_{2}^{(0)}$ are constant coefficients given by 


$$
\begin{aligned}
& M_{1}^{(0)}=-2 \pi^{2}\left[A_{1}+A_{2}\left(\frac{\sqrt{6 \pi} \Gamma\left(\frac{2}{3}\right)}{3 \Gamma\left(\frac{7}{6}\right)}+\sum_{m=1}^{\infty}\left(\frac{\sqrt{6} \Gamma\left(m+\frac{1}{2}\right) \Gamma\left(\frac{2 m}{3}+\frac{2}{3}\right)}{3 \Gamma(m+1) \Gamma\left(\frac{2 m}{3}+\frac{7}{6}\right)}-\frac{1}{m}\right)\right)\right] \\
& M_{2}^{(0)}=\sum_{p=1}^{\infty} \frac{\pi^{5 / 2} \Gamma\left(p+\frac{1}{2}\right)}{\Gamma(p+1)(4 p-3)}-\frac{\pi^{3}}{3}+2 \sqrt{6} \pi^{3} A_{2} .
\end{aligned}
$$

Finally, HSC can be obtained using Eqs. (12) and (60). According to Eq. (60), at high temperature, the term including $M_{2}^{(0)}$ is dominant indicating $V_{H T}^{(0)}$ is proportional to $l T$ and the term independent of $l T$ receives contribution from the full bulk background.

(b) Up to $\left(r_{c} / r_{*}\right)^{2}$ : We consider the terms including $L_{2}$ and $C_{2}$ which are the nonconformal effects at high temperature. Using (52) and (53), we have

$$
\frac{V_{\gamma_{A}}}{L^{2}}=l r_{*}^{3}\left(\sum_{p=1}^{\infty} \frac{\Gamma\left(p+\frac{1}{2}\right)}{\sqrt{\pi} \Gamma(p+1)(4 p-3)}\left(\frac{r_{h}}{r_{*}}\right)^{4 p}-\frac{1}{3}\right)+2 r_{*}^{2}\left(\mathcal{B}\left(\frac{r_{c}}{r_{*}}\right)^{2}-\mathcal{A}\right),
$$

where again the term $-1 / 3$ comes from $p=0$ term which is the divergent part and $\mathcal{B}$ is given by

$$
\begin{aligned}
\mathcal{B}= & \sum_{n=0}^{\infty} \sum_{m=0}^{\infty} \sum_{p=0}^{\infty} \frac{\Gamma\left(n+\frac{1}{2}\right) \Gamma\left(m+\frac{1}{2}\right) \Gamma\left(p+\frac{1}{2}\right)}{\pi^{3 / 2} \Gamma(n+1) \Gamma(m+1) \Gamma(p+1)}\left[\frac{3}{2}(2 n+1)\right. \\
& \times\left(\frac{1}{(6 n+4 m+6)(6 n+4 m+4 p+3)}-\frac{1}{(6 n+4 m+4)(6 n+4 m+4 p+1)}\right) \\
& \left.+\frac{2}{(6 n+4 m+4)(4 p-1)}-\frac{2}{(6 n+4 m+4)(6 n+4 m+4 p+3)}\right]\left(\frac{r_{h}}{r_{*}}\right)^{4(m+p)} .
\end{aligned}
$$

In order to study the convergence of the series in (63) at high temperature, $r_{*} \sim r_{h}$, we divide $\mathcal{B}$ into the two infinite series called $\mathcal{B}_{1}$ and $\mathcal{B}_{2}$ which is investigated respectively. First we study the behavior of $\mathcal{B}_{1}$ whose form is written by

$$
\begin{aligned}
\mathcal{B}_{1}= & \sum_{n=0}^{\infty} \sum_{m=0}^{\infty} \sum_{p=0}^{\infty} \frac{\Gamma\left(n+\frac{1}{2}\right) \Gamma\left(m+\frac{1}{2}\right) \Gamma\left(p+\frac{1}{2}\right)}{\pi^{3 / 2} \Gamma(n+1) \Gamma(m+1) \Gamma(p+1)} \frac{3}{2}(2 n+1) \\
& \times\left(\frac{1}{(6 n+4 m+6)(6 n+4 m+4 p+3)}-\frac{1}{(6 n+4 m+4)(6 n+4 m+4 p+1)}\right)\left(\frac{r_{h}}{r_{*}}\right)^{4(m+p)} \\
= & \sum_{n=0}^{\infty} \sum_{m=0}^{\infty}\left[\frac{3(2 n+1) \Gamma\left(n+\frac{1}{2}\right) \Gamma\left(m+\frac{1}{2}\right)}{4 \pi(3 n+2 m+3)(6 n+4 m+3) \Gamma(n+1) \Gamma(m+1)}{ }_{2} F_{1}\left(\frac{1}{2}, m+\frac{3 n}{2}+\frac{3}{4} ; m+\frac{3 n}{2}+\frac{7}{4} ;\left(\frac{r_{h}}{r_{*}}\right)^{4}\right)\right. \\
& \left.-\frac{3(2 n+1) \Gamma\left(n+\frac{1}{2}\right) \Gamma\left(m+\frac{1}{2}\right)}{4 \pi(3 n+2 m+2)(6 n+4 m+1) \Gamma(n+1) \Gamma(m+1)}{ }_{2} F_{1}\left(\frac{1}{2}, m+\frac{3 n}{2}+\frac{1}{4} ; m+\frac{3 n}{2}+\frac{5}{4} ;\left(\frac{r_{h}}{r_{*}}\right)^{4}\right)\right]\left(\frac{r_{h}}{r_{*}}\right)^{4 m},
\end{aligned}
$$

where in the last equation we sum over $p$. For large $n$ and $m$, there is an equal behavior regarding the two terms in (64) due to their functional form and both of them converge at high temperature, $r_{*} \sim r_{h}$. We are left to check the convergence of the remaining part of $\mathcal{B}$, called $\mathcal{B}_{2}$, which is given by the following expression 


$$
\begin{aligned}
& \mathcal{B}_{2}=\sum_{n=0}^{\infty} \sum_{m=0}^{\infty} \sum_{p=0}^{\infty} \frac{\Gamma\left(n+\frac{1}{2}\right) \Gamma\left(m+\frac{1}{2}\right) \Gamma\left(p+\frac{1}{2}\right)}{\pi^{3 / 2} \Gamma(n+1) \Gamma(m+1) \Gamma(p+1)}\left(\frac{2}{(6 n+4 m+4)(4 p-1)}\right. \\
& \left.-\frac{2}{(6 n+4 m+4)(6 n+4 m+4 p+3)}\right)\left(\frac{r_{h}}{r_{*}}\right)^{4(m+p)} \\
& =\sum_{m=0}^{\infty}\left(\frac{r_{h}}{r_{*}}\right)^{4 m}\left[\frac{\Gamma\left(m+\frac{1}{2}\right) \Gamma\left(\frac{2 m}{3}+\frac{3}{2}\right)}{84(4 m+3)(4 m+7)(4 m+11) \Gamma(m+1) \Gamma\left(\frac{2 m}{3}+1\right)}\right. \\
& \times{ }_{6} F_{5}\left(-\frac{1}{12}, \frac{1}{6}, \frac{1}{2}, \frac{5}{6}, \frac{m}{3}+\frac{1}{4}, \frac{m}{3}+\frac{3}{4} ; \frac{1}{3}, \frac{2}{3}, \frac{11}{12}, \frac{m}{3}+\frac{1}{2}, \frac{m}{3}+1 ;\left(\frac{r_{h}}{r_{*}}\right)^{12}\right) \\
& \times\left(-12936-12096 m-2688 m^{2}\right)+\frac{\Gamma\left(m+\frac{1}{2}\right) \Gamma\left(\frac{2 m}{3}+\frac{13}{6}\right)}{84(4 m+3)(4 m+7)(4 m+11) \Gamma(m+1) \Gamma\left(\frac{2 m}{3}+\frac{5}{3}\right)} \\
& \times{ }_{6} F_{5}\left(\frac{1}{4}, \frac{1}{2}, \frac{5}{6}, \frac{7}{6}, \frac{m}{3}+\frac{7}{12}, \frac{m}{3}+\frac{13}{12} ; \frac{2}{3}, \frac{5}{4}, \frac{4}{3}, \frac{m}{3}+\frac{5}{6}, \frac{m}{3}+\frac{4}{3} ;\left(\frac{r_{h}}{r_{*}}\right)^{12}\right)\left(\frac{r_{h}}{r_{*}}\right)^{4} \\
& \times\left(924+1568 m+448 m^{2}\right)+\frac{\Gamma\left(m+\frac{1}{2}\right) \Gamma\left(\frac{2 m}{3}+\frac{17}{6}\right)}{84(4 m+3)(4 m+7)(4 m+11) \Gamma(m+1) \Gamma\left(\frac{2 m}{3}+\frac{7}{3}\right)} \\
& \times{ }_{6} F_{5}\left(\frac{7}{12}, \frac{5}{6}, \frac{7}{6}, \frac{3}{2}, \frac{m}{3}+\frac{11}{12}, \frac{m}{3}+\frac{17}{12} ; \frac{4}{3}, \frac{19}{12}, \frac{5}{3}, \frac{m}{3}+\frac{7}{6}, \frac{m}{3}+\frac{5}{3} ;\left(\frac{r_{h}}{r_{*}}\right)^{12}\right)\left(\frac{r_{h}}{r_{*}}\right)^{8} \\
& \left.\times\left(189+360 m+144 m^{2}\right)\right] \text {, }
\end{aligned}
$$

where in the last equation we sum over $n$ and $p$. There exist three different types of behavior for large $m$ in (65). There are specific terms whose large $m$ behavior goes as $\sim m^{-3}\left(r_{h} / r_{*}\right)^{4 m}$ and $\sim m^{-2}\left(r_{h} / r_{*}\right)^{4 m}$ which are clearly convergent at $r_{*}=r_{h}$, by isolation of the term corresponding to $m=0$ of the series. However, there are other terms which go as $\sim m^{-1}\left(r_{h} / r_{*}\right)^{4 m}$ and hence they are divergent at $r_{*}=r_{h}$. In order to get convergent series we should extract the divergence piece. Similar to the previous calculation of the zeroth order of $r_{c} / r_{*}$, we finally get

$$
\mathcal{B}=B_{1}+B_{2} \ln (4 \epsilon)+\mathcal{O}(\epsilon),
$$

where $B_{1}$ and $B_{2}$ are constant coefficients given in Appendix $\mathrm{B}$ and $\ln (4 \epsilon)$ by considering the second order of $r_{c} / r_{*}$, is a function of $r_{h}$ and $l$ obtained in Appendix B. Using (62) and (66) we have

$$
\begin{aligned}
V_{H T}^{(2)}\left(l \Lambda_{c}, l T\right) \equiv & \frac{V_{\gamma_{A}}\left(l, l \Lambda_{c}, l T\right)}{L^{2} \Lambda_{c} T} \\
= & \frac{1}{l^{2} \Lambda_{c} T}\left[\left(M_{1}^{(0)}+l T M_{2}^{(0)}\right)(l T)^{2}\right. \\
& \left.+\left(M_{1}^{(2)}+l T M_{2}^{(2)}\right)\left(l \Lambda_{c}\right)^{2}\right]
\end{aligned}
$$

where $V_{H T}^{(2)}$ is dimensionless volume up to the $\left(r_{c} / r_{*}\right)^{2}$ at high temperature, $M_{1}^{(2)}$ and $M_{2}^{(2)}$ are constant coefficients given by

$$
\begin{aligned}
M_{1}^{(2)}=B_{1} & +B_{2}\left(\frac{\sqrt{6 \pi} \Gamma\left(\frac{2}{3}\right)}{3 \Gamma\left(\frac{7}{6}\right)}+\sum_{m=1}^{\infty}\left(\frac{\sqrt{6} \Gamma\left(m+\frac{1}{2}\right) \Gamma\left(\frac{2 m}{3}+\frac{2}{3}\right)}{3 \Gamma(m+1) \Gamma\left(\frac{2 m}{3}+\frac{7}{6}\right)}-\frac{1}{m}\right)\right) \\
& -A_{2} \sqrt{6} \sum_{n=0}^{\infty}\left[\frac{3(2 n+1) \Gamma\left(n+\frac{1}{2}\right)}{2(3 n+2) \sqrt{\pi} \Gamma(n+1)}{ }_{2} F_{1}\left(\frac{1}{2}, \frac{3 n}{2}+1 ; \frac{3 n}{2}+2 ; 1\right)\right. \\
& \left.-\frac{(2 n+1) \Gamma\left(n+\frac{1}{2}\right)}{2(n+1) \sqrt{\pi} \Gamma(n+1)}{ }_{2} F_{1}\left(\frac{1}{2}, \frac{3 n}{2}+\frac{3}{2} ; \frac{3 n}{2}+\frac{5}{2} ; 1\right)\right] \\
M_{2}^{(2)}= & -\pi \sqrt{6} B_{2} .
\end{aligned}
$$



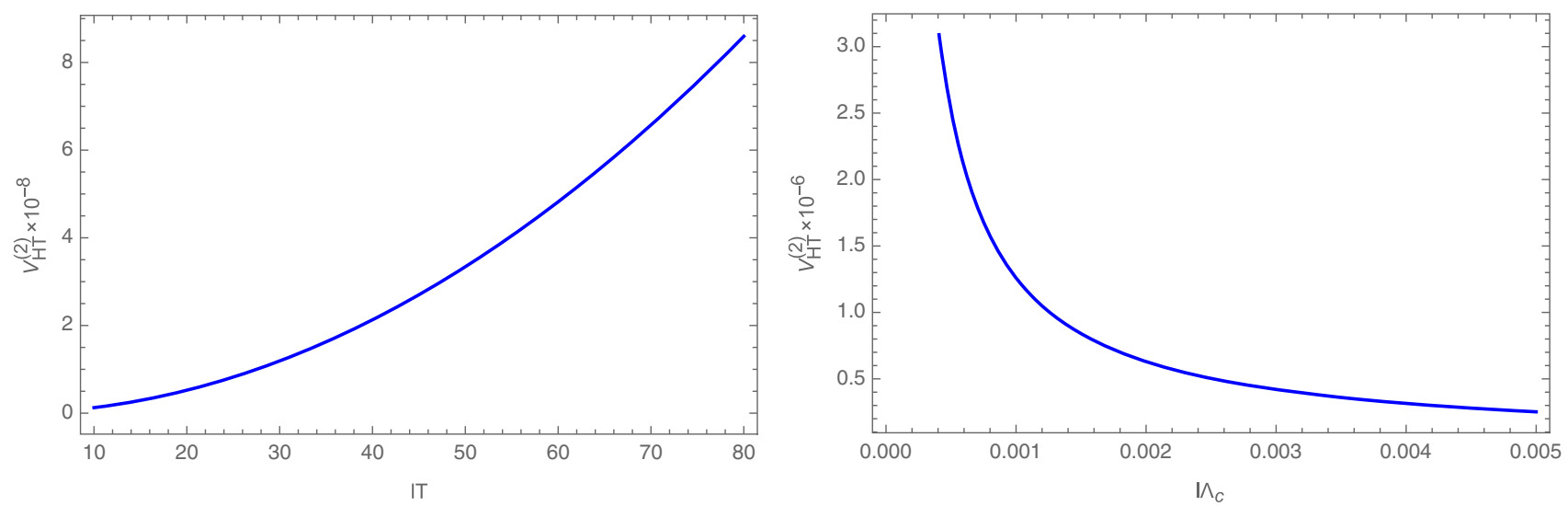

FIG. 4. Left: The dimensionless volume $V_{H T}^{(2)}$ as a function of $l T$ for fixed $l \Lambda_{c}=0.0001$, in the high temperature limit. The curve is described by $V_{H T}^{(2)}=\frac{M_{2}^{(0)}}{l \Lambda_{c}}(l T)^{2}$. Right: $V_{H}^{(2)}$ as a function of $l \Lambda_{c}$ for fixed $l T=10$ in the high temperature limit. The curve is described by $V_{H T}^{(2)}=\frac{1}{l \Lambda_{c}} \frac{M_{1}^{(0)}}{l T}$.

The terms including $M_{1}^{(0)}$ and $M_{2}^{(0)}$ come from the order $\left(r_{c} / r_{*}\right)^{0}$ which appeared in Eq. (60) and terms including $M_{1}^{(2)}$ and $M_{2}^{(2)}$ are corrections due to the nonconformal and thermal effects. Again HSC can be obtained using Eqs. (12) and (67). From Eq. (67), it is seen that if we fix $l \Lambda_{c}$ and increase $l T$, the $V_{H T}^{(2)}$ will increase. This can be better observed from the left panel of Fig. 4, where we plot $V_{H T}^{(2)}$ as a function of $l T$ for fixed $l \Lambda_{c}$. This behavior is the opposite of the behavior of low temperature case, left panel of Fig. 2. On the other side, if one fixes $l T$ and increases $l \Lambda_{c}$, then the $V_{H T}^{(2)}$ will decrease, the right panel of Fig. 4 where we plot $V_{H T}^{(2)}$ as a function of $l \Lambda_{c}$ for fixed $l T$. This behavior is the same as the behavior of the low temperature case, right panel of Fig. 2. We would like to compare HSC in the low and high temperature limit dropping the nonconformal effect. To do so, we need to take the limit $l \Lambda_{c} \rightarrow 0$ of Eqs. (67) and (48). The former limit is just the Eq. (60) and the later one has the following expression

$$
\frac{V_{\gamma_{A}}}{L^{2} T^{2}}=\frac{1}{(l T)^{2}}\left(\bar{w}_{1}+\bar{\omega}_{1}(l T)^{4}\right),
$$

where the left-hand side is the conformal dimensionless volume in the low temperature limit. Unlike Eq. (60), we do not obtain a linear relation in terms of $l T$ and it is observed that $l T$ has inverse effect on the mentioned subregion volume. Note that, in Sec. IV B 2 and V B 2, we use expression $r_{*}=r_{h}(1+\epsilon), \epsilon \ll 1$ to investigate the high temperature behavior of the meson potential energy and HSC. To get analytical results, we do the calculations up to order $\ln (4 \epsilon)$ for HSC, while the meson potential energy becomes zero up to that order.

\section{CONCLUSION}

In this paper, we study zero temperature and finite temperature potential energy and HSC of a probe meson using AdS/CFT correspondence in a nonconformal model. We develop a systematic expansion for those nonlocal observables and focus on the high energy limit, $r_{c} \ll r_{*}$ or $l \Lambda_{c} \ll 1$, leading to the analytical expressions in low and high temperature limits. The length of quark and antiquark pair is identified as the subregion length and we hence study the meson subregion complexity in the underlying model. In the particular regime of the model parameter, fixed $l \Lambda_{c}$, at leading order we find an interesting linear relation between dimensionless meson potential energy and dimensionless volume; that is the less binding meson state needs less information to be specified and vice versa at both zero and low temperatures. In zero and low temperature limits, nonconformal corrections decrease meson potential energy and increase HSC. At the low temperature limit, thermal corrections decrease meson potential energy and do not have a specific effect on HSC. However, at leading order, nonconformal and thermal effects have an equal effect on meson potential energy and HSC, implying a decreasing impact on them. At high temperature, thermal effects increase HSC, while at leading order nonconformal effects decrease it. Furthermore, in the high temperature limit, meson potential energy becomes zero. Near the transition point our calculation shows that less information is needed to specify the meson state at zero temperature. Hence, one can say that the meson state at zero temperature is more desirable. Several problems arise which we leave for further investigations:

(a) It would be interesting to study analytically, by using such systematic expansion, the relation between meson potential and subregion complexity using action prescription for nonconformal theories and to compare it to the volume prescription. It is interesting to check whether such linear relation exists for action prescription. 
(b) There are other quantum information quantities such as purification and fidelity and one can analytically study their relations with meson binding in nonconformal theories in order to establish the powerful properties of these quantities and match them with holographic conjectures in such non-conformal theories.

\section{APPENDIX A: POTENTIAL ENERGY}

(a) Zero temperature: In Sec. IV A 1, we reach Eq. (24) for the dimensionless meson potential energy $\tilde{V}_{q \bar{q}}$ at zero temperature in the high energy limit $r_{c} \ll r_{*}$. In the following we write the full calculations. Using Eq. (21) and keeping up to $\left(r_{c} / r_{*}\right)^{4}$, we obtain

$$
l\left(r_{*}\right)=\frac{2}{r_{*}} \sum_{n=0}^{\infty} \frac{\Gamma\left(n+\frac{1}{2}\right)}{\sqrt{\pi} \Gamma(n+1)} \int_{0}^{1} u^{4 n+2}\left[1+(2 n+1)\left(1-u^{2}\right)\left(\frac{r_{c}}{r_{*}}\right)^{2}+\frac{1}{2}(2 n+1)^{2}\left(1-u^{2}\right)^{2}\left(\frac{r_{c}}{r_{*}}\right)^{4}\right] d u .
$$

Integrating the above equation, we have an expression for $l$ as a function of $r_{*}$

$$
l\left(r_{*}\right)=\frac{2}{r_{*}}\left[a_{1}+a_{2}\left(\frac{r_{c}}{r_{*}}\right)^{2}+a_{3}\left(\frac{r_{c}}{r_{*}}\right)^{4}\right], \quad a_{1}, a_{2}, a_{3}>0,
$$

where constant coefficients are given by

$$
\begin{aligned}
a_{1} & \equiv \sum_{n=0}^{\infty} \frac{\Gamma\left(n+\frac{1}{2}\right)}{\sqrt{\pi} \Gamma(n+1)(4 n+3)}=\frac{\sqrt{\pi} \Gamma\left(\frac{7}{4}\right)}{3 \Gamma\left(\frac{5}{4}\right)}, \\
a_{2} & \equiv \sum_{n=0}^{\infty} \frac{2 \Gamma\left(n+\frac{1}{2}\right)(2 n+1)}{\sqrt{\pi} \Gamma(n+1)(4 n+3)(4 n+5)}=\frac{2}{15} \sqrt{\frac{2}{\pi}}\left[5 \Gamma\left(\frac{3}{4}\right) \Gamma\left(\frac{7}{4}\right)-\Gamma\left(\frac{1}{4}\right) \Gamma\left(\frac{9}{4}\right)\right]+\frac{2}{63}{ }_{3} F_{2}\left(\frac{3}{2}, \frac{7}{4}, \frac{9}{4} ; \frac{11}{4}, \frac{13}{4} ; 1\right), \\
a_{3} & \equiv \sum_{n=0}^{\infty} \frac{4 \Gamma\left(n+\frac{1}{2}\right)(2 n+1)^{2}}{\sqrt{\pi} \Gamma(n+1)(4 n+3)(4 n+5)(4 n+7)} \\
& =\frac{4 \sqrt{\pi}}{15}\left(\frac{\Gamma\left(\frac{11}{4}\right)}{\Gamma\left(\frac{5}{4}\right)}-\frac{\Gamma\left(\frac{9}{4}\right)}{\Gamma\left(\frac{3}{4}\right)}\right)+\frac{4}{693}\left[2_{4} F_{3}\left(\frac{4}{3}, \frac{7}{4}, 2, \frac{9}{4} ; 1, \frac{13}{4}, \frac{15}{4} ; 1\right)-7_{3} F_{2}\left(\frac{3}{2}, \frac{9}{4}, \frac{11}{4} ; \frac{13}{4}, \frac{15}{4} ; 1\right)\right] .
\end{aligned}
$$

In high energy limit, the corrections to the pure AdS are small and then one can solve Eq. (A2) perturbatively for $r_{*}$

$$
r_{*}(l)=\frac{1}{l}\left(\bar{a}_{1}+\frac{\bar{a}_{2}}{2}\left(l \Lambda_{c}\right)^{2}+\frac{\bar{a}_{3}}{4}\left(l \Lambda_{c}\right)^{4}\right) \quad \bar{a}_{1}, \bar{a}_{2}>0, \quad \bar{a}_{3}<0,
$$

where $\bar{a}_{1}, \bar{a}_{2}$ and $\bar{a}_{3}$ are constant coefficients given by

$$
\begin{aligned}
\bar{a}_{1}= & \frac{2 \sqrt{\pi} \Gamma\left(\frac{7}{4}\right)}{3 \Gamma\left(\frac{5}{4}\right)}, \\
\bar{a}_{2}= & \frac{1}{560 \sqrt{2} \pi^{3 / 2} \Gamma\left(\frac{7}{4}\right)^{2}}\left\{315 \pi^{2}+\Gamma\left(\frac{1}{4}\right)^{2}\left[-42 \Gamma\left(\frac{1}{4}\right) \Gamma\left(\frac{9}{4}\right)+5 \sqrt{2 \pi}_{3} F_{2}\left(\frac{3}{2}, \frac{7}{4}, \frac{9}{4} ; \frac{11}{4}, \frac{13}{4} ; 1\right)\right]\right\}, \\
\bar{a}_{3}= & \frac{1}{574013440 \pi^{9 / 2} \Gamma\left(\frac{7}{4}\right)^{4}}\left\{-82657935360 \sqrt{2} \Gamma\left(\frac{5}{4}\right)^{10}+1968046080 \sqrt{\pi} \Gamma\left(\frac{1}{4}\right) \Gamma\left(\frac{5}{4}\right)_{3}^{7} F_{2}\left(\frac{3}{2}, \frac{7}{4}, \frac{9}{4} ; \frac{11}{4}, \frac{13}{4} ; 1\right)\right. \\
& +715 \sqrt{\pi}\left[\Gamma\left(\frac{1}{4}\right)^{6}\left(3969 \pi-32_{3} F_{2}\left(\frac{3}{2}, \frac{7}{4}, \frac{9}{4} ; \frac{11}{4}, \frac{13}{4} ; 1\right)^{2}\right]\right. \\
& +2016 \pi^{5 / 2} \Gamma\left(\frac{1}{4}\right)^{4}\left[1430_{3} F_{2}\left(\frac{3}{2}, \frac{7}{4}, \frac{9}{4} ; \frac{11}{4}, \frac{13}{4} ; 1\right)+910_{3} F_{2}\left(\frac{3}{2}, \frac{9}{4}, \frac{11}{4} ; \frac{13}{4}, \frac{15}{4} ; 1\right)\right. \\
& \left.\left.-63_{3} F_{2}\left(\frac{5}{2}, \frac{11}{4}, \frac{13}{4} ; \frac{17}{4}, \frac{19}{4} ; 1\right)\right]\right\} .
\end{aligned}
$$

We do the same calculation for $\tilde{V}_{q \bar{q}}$, Eq. (22), and expanding it to $\left(r_{c} / r_{*}\right)^{4}$ we get 
$V_{q \bar{q}}\left(r_{*}\right)=\frac{r_{*}}{\pi \alpha^{\prime}} \sum_{n=0}^{\infty} \frac{\Gamma\left(n+\frac{1}{2}\right)}{\sqrt{\pi} \Gamma(n+1)} \int_{\delta}^{1} u^{4 n-2}\left[1+\left(2 n+(1-2 n) u^{2}\right)\left(\frac{r_{c}}{r_{*}}\right)^{2}+\frac{1}{2}\left(2 n+(1-2 n) u^{2}\right)^{2}\left(\frac{r_{c}}{r_{*}}\right)^{4}\right] d u$.

By integration of Eq. (A6) and considering finite terms (the terms which do not include $\delta$ ) we reach

$$
V_{q \bar{q}}\left(r_{*}\right)=\frac{r_{*}}{\pi \alpha^{\prime}}\left[b_{1}+b_{2}\left(\frac{r_{c}}{r_{*}}\right)^{2}+b_{3}\left(\frac{r_{c}}{r_{*}}\right)^{4}\right], \quad b_{1}<0, \quad b_{2}, b_{3}>0,
$$

where constant coefficients $b_{1}, b_{2}$ and $b_{3}$ are given by

$$
\begin{aligned}
b_{1} & \equiv \sum_{n=0}^{\infty} \frac{\Gamma\left(n+\frac{1}{2}\right)}{\sqrt{\pi} \Gamma(n+1)(4 n-1)}=-\frac{\sqrt{\pi} \Gamma\left(\frac{3}{4}\right)}{\Gamma\left(\frac{1}{4}\right)}, \\
b_{2} & \equiv \sum_{n=0}^{\infty} \frac{\Gamma\left(n+\frac{1}{2}\right)(1-8 n)}{\sqrt{\pi} \Gamma(n+1)\left(1-16 n^{2}\right)}=\frac{\Gamma\left(\frac{1}{4}\right)^{2}-\Gamma\left(-\frac{1}{4}\right) \Gamma\left(\frac{3}{4}\right)}{8 \sqrt{2 \pi}}+\frac{4}{15}{ }_{3} F_{2}\left(\frac{3}{4}, \frac{5}{4}, \frac{3}{2} ; \frac{7}{4}, \frac{9}{4} ; 1\right), \\
b_{3} & \equiv \sum_{n=0}^{\infty} \frac{\Gamma\left(n+\frac{1}{2}\right)\left(1+8 n-80 n^{2}\right)}{\sqrt{\pi} \Gamma(n+1)\left(6+8 n-96 n^{2}-128 n^{3}\right)} \\
& =\frac{\Gamma\left(\frac{5}{4}\right)^{2}}{2 \sqrt{2 \pi}}+\frac{3 \sqrt{\pi} \Gamma\left(\frac{7}{4}\right)}{\Gamma\left(\frac{1}{4}\right)}-\frac{9}{35^{3}} F_{2}\left(\frac{5}{4}, \frac{3}{2}, \frac{7}{4} ; \frac{9}{4}, \frac{11}{4} ; 1\right)+\frac{10}{231_{3}} F_{2}\left(\frac{7}{4}, \frac{9}{4}, \frac{5}{2} ; \frac{13}{4}, \frac{15}{4} ; 1\right) .
\end{aligned}
$$

Using Eq. (A4) and substituting $r_{c}=\Lambda_{c} / \sqrt{2}$, we reach the final result

$$
\tilde{V}_{q \bar{q}}\left(l \Lambda_{c}\right) \equiv \frac{\alpha^{\prime} V_{q \bar{q}}\left(l, l \Lambda_{c}\right)}{\Lambda_{c}}=\frac{1}{l \Lambda_{c}}\left(\bar{b}_{1}+\frac{\bar{b}_{2}}{2}\left(l \Lambda_{c}\right)^{2}+\frac{\bar{b}_{3}}{4}\left(l \Lambda_{c}\right)^{4}\right), \quad \bar{b}_{1}<0, \quad \bar{b}_{2}, \bar{b}_{3}>0,
$$

where $\tilde{V}_{q \bar{q}}$ is dimensionless meson potential energy at zero temperature. All the constant coefficients are summarized in Table I.

$$
\begin{aligned}
\bar{b}_{1}= & -\frac{2 \Gamma\left(\frac{3}{4}\right) \Gamma\left(\frac{7}{4}\right)}{3 \Gamma\left(\frac{1}{4}\right) \Gamma\left(\frac{5}{4}\right)}, \\
\bar{b}_{2}= & \frac{1}{8960 \pi \Gamma\left(\frac{7}{4}\right)^{2}}\left\{525 \Gamma\left(\frac{1}{4}\right)^{2}+315 \Gamma\left(\frac{-1}{4}\right) \Gamma\left(\frac{3}{4}\right)\right. \\
& \left.+16 \sqrt{2 \pi}\left[42_{3} F_{2}\left(\frac{3}{4}, \frac{5}{4}, \frac{3}{2} ; \frac{7}{4}, \frac{9}{4} ; 1\right)-5_{3} F_{2}\left(\frac{3}{2}, \frac{7}{4}, \frac{9}{4} ; \frac{11}{4}, \frac{13}{4} ; 1\right)\right]\right\}, \\
\bar{b}_{3}= & \frac{1}{4592107520 \pi^{4} \Gamma\left(\frac{7}{4}\right)^{4}}\left\{\Gamma ( \frac { 1 } { 4 } ) ^ { 2 } \left[-630630 \pi^{2}\left(87 \Gamma\left(\frac{1}{4}\right)^{2}-896 \Gamma\left(\frac{7}{4}\right)^{2}\right)\right.\right. \\
& +3003 \Gamma\left(\frac{1}{4}\right)^{4}\left[735 \Gamma\left(\frac{1}{4}\right)^{2}+8 \sqrt{2 \pi}\left(84_{3} F_{2}\left(\frac{3}{4}, \frac{5}{4}, \frac{3}{2} ; \frac{7}{4}, \frac{9}{4} ; 1\right)-55_{3} F_{2}\left(\frac{3}{2}, \frac{7}{4}, \frac{9}{4} ; \frac{11}{4}, \frac{13}{4} ; 1\right)\right)\right] \\
& -73216 \pi \Gamma\left(\frac{1}{4}\right)_{3}^{2} F_{2}\left(\frac{3}{2}, \frac{7}{4}, \frac{9}{4} ; \frac{11}{4}, \frac{13}{4} ; 1\right)\left[21_{3} F_{2}\left(\frac{3}{4}, \frac{5}{4}, \frac{3}{2} ; \frac{7}{4}, \frac{9}{4} ; 1\right)-5_{3} F_{2}\left(\frac{3}{2}, \frac{7}{4}, \frac{9}{4} ; \frac{11}{4}, \frac{13}{4} ; 1\right)\right] \\
& -4032 \sqrt{2} \pi^{5 / 2}\left[12012_{3} F_{2}\left(\frac{3}{4}, \frac{5}{4}, \frac{3}{2} ; \frac{7}{4}, \frac{9}{4} ; 1\right)+11583_{3} F_{2}\left(\frac{5}{4}, \frac{3}{2}, \frac{7}{4} ; \frac{9}{4}, \frac{11}{4} ; 1\right)-5005 F_{3} F_{2}\left(\frac{3}{2}, \frac{7}{4}, \frac{9}{4} ; \frac{11}{4}, \frac{13}{4} ; 1\right)\right. \\
& \left.\left.\left.+3640_{3} F_{2}\left(\frac{3}{2}, \frac{9}{4}, \frac{5}{2} ; \frac{13}{4}, \frac{15}{4} ; 1\right)+1950_{3} F_{2}\left(\frac{7}{4}, \frac{9}{4}, \frac{5}{2} ; \frac{13}{4}, \frac{15}{4} ; 1\right)+252_{3} F_{2}\left(\frac{5}{2}, \frac{11}{4}, \frac{13}{4} ; \frac{17}{4}, \frac{19}{4} ; 1\right)\right]\right]\right\}
\end{aligned}
$$

TABLE I. Numerical constant coefficients for potential calculation.

\begin{tabular}{lll}
\hline \hline$a_{1}=0.59907$ & $a_{2}=0.355979$ & $a_{3}=0.214869$ \\
$\bar{a}_{1}=1.19814$ & $\bar{a}_{2}=0.495952$ & $\bar{a}_{3}=-0.202051$ \\
$b_{1}=-0.59907$ & $b_{2}=1.66701$ & $b_{3}=0.813939$ \\
$\bar{b}_{1}=-0.228473$ & $\bar{b}_{2}=0.348301$ & $\bar{b}_{3}=0.00584084$ \\
\hline \hline
\end{tabular}


(b) Low temperature: In Sec. IV B 1, Eq. (30) is reached for the dimensionless meson potential energy $\hat{V}_{q \bar{q}}$ at low temperature $l T \ll 1$ in the high energy limit $r_{c} \ll r_{*}$. In the following we review the details of the computations. Using (27) and expanding it up to $\left(r_{c} / r_{*}\right)^{4}$ and $\left(r_{h} / r_{*}\right)^{4}$ we get

$$
\begin{aligned}
l\left(r_{*}\right)= & \frac{2}{r_{*}} \sum_{n=0}^{\infty} \frac{\Gamma\left(n+\frac{1}{2}\right)}{\sqrt{\pi} \Gamma(n+1)} \int_{0}^{1} u^{4 n+2}\left\{1+\left((n+1) u^{4 n+4}-\left(n+\frac{1}{2}\right)\right)\left(\frac{r_{h}}{r_{*}}\right)^{4}\right. \\
& +\left[(2 n+1)\left(1-u^{2}\right)+\left((2 n+1)\left(1-u^{2}\right)\left((n+1) u^{4 n+4}-\left(n+\frac{1}{2}\right)\right)\right)\left(\frac{r_{h}}{r_{*}}\right)^{4}\right]\left(\frac{r_{c}}{r_{*}}\right)^{2} \\
& +\frac{1}{2}\left[(2 n+1)^{2}\left(1-u^{2}\right)^{2}+\left((2 n+1)^{2}\left(1-u^{2}\right)^{2}\left((n+1) u^{4 n+4}-\left(n+\frac{1}{2}\right)\right)\left(\frac{r_{h}}{r_{*}}\right)^{4}\right]\left(\frac{r_{c}}{r_{*}}\right)^{4}\right\} d u .
\end{aligned}
$$

Integrating Eq. (A11), we obtain $l$ in terms of $r_{*}$

$$
l\left(r_{*}\right)=\frac{2}{r_{*}}\left[a_{1}+\alpha_{1}\left(\frac{r_{h}}{r_{*}}\right)^{4}+\left(a_{2}+\alpha_{2}\left(\frac{r_{h}}{r_{*}}\right)^{4}\right)\left(\frac{r_{c}}{r_{*}}\right)^{2}+\left(a_{3}+\alpha_{3}\left(\frac{r_{h}}{r_{*}}\right)^{4}\right)\left(\frac{r_{c}}{r_{*}}\right)^{4}\right], \quad \alpha_{1}, \alpha_{2}, \quad \alpha_{3}<0
$$

where $\alpha_{1}, \alpha_{2}, \alpha_{3}$ are given by

$$
\begin{aligned}
\alpha_{1} \equiv & -\sum_{n=0}^{\infty} \frac{\Gamma\left(n+\frac{1}{2}\right)(4 n+1)}{\sqrt{\pi} \Gamma(n+1)\left(32 n^{2}+80 n+42\right)}=-\frac{\sqrt{\pi}\left(11 \Gamma\left(\frac{11}{4}\right)+12 \Gamma\left(\frac{15}{4}\right)\right)}{924 \Gamma\left(\frac{9}{4}\right)}, \\
\alpha_{2} \equiv & -\sum_{n=0}^{\infty} \frac{3 \Gamma\left(n+\frac{1}{2}\right)(2 n+1)\left(16 n^{2}+32 n+11\right)}{\sqrt{\pi} \Gamma(n+1)(4 n+3)(4 n+5)(4 n+7)(4 n+9)}=\frac{\frac{1291}{84} \Gamma\left(\frac{5}{4}\right)^{2}-\frac{71}{9} \Gamma\left(\frac{7}{4}\right)^{2}}{\sqrt{2 \pi}} \\
& -\frac{67}{286}{ }_{3} F_{2}\left(\frac{3}{2}, \frac{11}{4}, \frac{13}{4} ; \frac{15}{4}, \frac{17}{4} ; 1\right)-\frac{6}{187}{ }_{3} F_{2}\left(\frac{5}{2}, \frac{11}{4}, \frac{17}{4} ; \frac{19}{4}, \frac{21}{4} ; 1\right) \\
& +\frac{6}{221}{ }_{3} F_{2}\left(\frac{5}{2}, \frac{13}{4}, \frac{15}{4} ; \frac{19}{4}, \frac{21}{4} ; 1\right)-\frac{16}{3003}{ }_{5} F_{4}\left(\frac{3}{2}, \frac{7}{4}, 2,2, \frac{9}{4} ; 1,1, \frac{15}{4}, \frac{17}{4} ; 1\right), \\
\alpha_{3} \equiv & -\sum_{n=0}^{\infty} \frac{2 \Gamma\left(n+\frac{1}{2}\right)\left((2 n+1)^{2}\left(80 n^{2}+184 n+69\right)\right)}{\sqrt{\pi} \Gamma(n+1)(4 n+3)(4 n+5)(4 n+7)(4 n+9)(4 n+11)} \\
= & -\frac{2}{135135}\left[897{ }_{3} F_{2}\left(\frac{1}{2}, \frac{3}{4}, \frac{5}{4} ; \frac{13}{4}, \frac{15}{4} ; 1\right)+230_{3} F_{2}\left(\frac{3}{2}, \frac{7}{4}, \frac{9}{4} ; \frac{17}{4}, \frac{19}{4} ; 1\right)\right. \\
& +546_{4} F_{3}\left(\frac{3}{2}, \frac{7}{4}, 2, \frac{9}{4} ; 1, \frac{17}{4}, \frac{19}{4} ; 1\right)+528_{5} F_{4}\left(\frac{3}{2}, \frac{7}{4}, 2,2, \frac{9}{4} ; 1,1, \frac{17}{4}, \frac{19}{4} ; 1\right) \\
& \left.+160_{6} F_{5}\left(\frac{3}{2}, \frac{7}{4}, 2,2,2, \frac{9}{4} ; 1,1,1, \frac{17}{4}, \frac{19}{4} ; 1\right)\right] .
\end{aligned}
$$

In low temperature and high energy limits, the corrections to pure AdS and nonconformal terms are small and hence can be computed perturbatively. Solving Eq. (A12) order by order for $r_{*}$

$$
r_{*}(l)=\frac{1}{l}\left(\bar{a}_{1}+\frac{\bar{a}_{2}}{2}\left(l \Lambda_{c}\right)^{2}+\frac{\bar{a}_{3}}{4}\left(l \Lambda_{c}\right)^{4}+\bar{a}_{4}(l T)^{4}\right), \quad \bar{a}_{1}, \bar{a}_{2}>0, \quad \bar{a}_{3}, \bar{a}_{4}<0
$$

where $\bar{a}_{4}$ is given by

$$
\bar{a}_{4}=-\frac{27 \Gamma\left(\frac{5}{4}\right)^{4}}{2464\left(\pi^{3 / 2} \Gamma\left(\frac{7}{4}\right)^{4} \Gamma\left(\frac{9}{4}\right)\right)}\left[11 \Gamma\left(\frac{11}{4}\right)+12 \Gamma\left(\frac{15}{4}\right)\right]
$$

We use Eq. (28) and expand it up to $\left(r_{c} / r_{*}\right)^{4}$ and $\left(r_{h} / r_{*}\right)^{4}$. Then we have 


$$
\begin{aligned}
V_{q \bar{q}}\left(r_{*}\right)= & \frac{r_{*}}{\pi \alpha^{\prime}} \sum_{n=0}^{\infty} \frac{\Gamma\left(n+\frac{1}{2}\right)}{\sqrt{\pi} \Gamma(n+1)} \int_{\delta}^{1} u^{4 n-2}\left\{1+n\left(u^{4}-1\right)\left(\frac{r_{h}}{r_{*}}\right)^{4}\right. \\
& +\left[2 n+(1-2 n) u^{2}+\left(n\left(2 n+(1-2 n) u^{2}\right)\left(u^{4}-1\right)\right)\left(\frac{r_{h}}{r_{*}}\right)^{4}\right]\left(\frac{r_{c}}{r_{*}}\right)^{2} \\
& \left.+\frac{1}{2}\left[\left(2 n+(1-2 n) u^{2}\right)^{2}+\left(n\left(2 n+(1-2 n) u^{2}\right)^{2}\left(u^{4}-1\right)\right)\left(\frac{r_{h}}{r_{*}}\right)^{4}\right]\left(\frac{r_{c}}{r_{*}}\right)^{4}\right\} d u .
\end{aligned}
$$

Integrating Eq. (A16) and keep the finite terms (terms without $\delta$ ),

$$
V_{q \bar{q}}\left(r_{*}\right)=\frac{r_{*}}{\pi \alpha^{\prime}}\left[b_{1}+\beta_{1}\left(\frac{r_{h}}{r_{*}}\right)^{4}+\left(b_{2}+\beta_{2}\left(\frac{r_{h}}{r_{*}}\right)^{4}\right)\left(\frac{r_{c}}{r_{*}}\right)^{2}+\left(b_{2}+\beta_{3}\left(\frac{r_{h}}{r_{*}}\right)^{4}\right)\left(\frac{r_{c}}{r_{*}}\right)^{4}\right], \quad \beta_{1}, \beta_{2}, \beta_{3}<0
$$

where $\beta_{1}, \beta_{2}$ and $\beta_{3}$ are constant coefficients given by

$$
\begin{aligned}
\beta_{1} \equiv & -\sum_{n=0}^{\infty} \frac{4 n \Gamma\left(n+\frac{1}{2}\right)}{\sqrt{\pi} \Gamma(n+1)\left(16 n^{2}+8 n-3\right)}=-\frac{2 \sqrt{\pi} \Gamma\left(\frac{11}{4}\right)}{21 \Gamma\left(\frac{5}{4}\right)}, \\
\beta_{2} \equiv & -\sum_{n=0}^{\infty} \frac{12 n \Gamma\left(n+\frac{1}{2}\right)\left(16 n^{2}+8 n-1\right)}{\sqrt{\pi} \Gamma(n+1)(4 n-1)(4 n+1)(4 n+3)(4 n+5)}=\frac{5 \Gamma\left(-\frac{1}{4}\right) \Gamma\left(\frac{11}{4}\right)-7 \Gamma\left(-\frac{3}{4}\right) \Gamma\left(\frac{13}{4}\right)}{30 \sqrt{2 \pi}} \\
& -\frac{1}{9}{ }_{3} F_{2}\left(\frac{3}{2}, \frac{7}{4}, \frac{9}{4} ; \frac{11}{4}, \frac{13}{4} ; 1\right)-\frac{36_{3} F_{2}\left(\frac{7}{4}, \frac{5}{2}, \frac{13}{4} ; \frac{15}{4}, \frac{17}{4} ; 1\right)-28_{3} F_{2}\left(\frac{9}{4}, \frac{5}{2}, \frac{11}{4} ; \frac{15}{4}, \frac{17}{4} ; 1\right)}{1001} \\
& -\frac{32}{315}{ }_{5} F_{4}\left(\frac{3}{4}, \frac{5}{4}, \frac{3}{2}, 2,2 ; 1,1, \frac{11}{4}, \frac{13}{4} ; 1\right), \\
\beta_{3} \equiv & -\sum_{n=0}^{\infty} \frac{2 n \Gamma\left(n+\frac{1}{2}\right)\left(4 n\left(176 n^{2}+140 n-17\right)-5\right)}{\sqrt{\pi} \Gamma(n+1)(4 n-1)(4 n+1)(4 n+3)(4 n+5)(4 n+7)} \\
= & -\frac{1}{10395}\left[5_{3} F_{2}\left(\frac{3}{4}, \frac{5}{4}, \frac{3}{2} ; \frac{13}{4}, \frac{15}{4} ; 1\right)+68_{4} F_{3}\left(\frac{3}{4}, \frac{5}{4}, \frac{3}{2}, 2 ; 1, \frac{13}{4}, \frac{15}{4} ; 1\right)\right. \\
& \left.-560_{5} F_{4}\left(\frac{3}{4}, \frac{5}{4}, \frac{3}{2}, 2,2 ; 1,1, \frac{13}{4}, \frac{15}{4} ; 1\right)-704_{6} F_{5}\left(\frac{3}{4}, \frac{5}{4}, \frac{3}{2}, 2,2,2 ; 1,1,1, \frac{13}{4}, \frac{15}{4} ; 1\right)\right] .
\end{aligned}
$$

Using Eq. (A14) and substituting $r_{c}=\Lambda_{c} / \sqrt{2}$ and $r_{h}=\pi T$ we reach the final result

$$
\begin{aligned}
\hat{V}_{q \bar{q}}\left(l \Lambda_{c}, l T\right) & \equiv \frac{\alpha^{\prime} V_{q \bar{q}}\left(l, l \Lambda_{c}, l T\right)}{\Lambda_{c}^{\frac{1}{2}} T^{\frac{1}{2}}} \\
& =\frac{1}{l \Lambda_{c}^{\frac{1}{2}} T^{\frac{1}{2}}}\left[\bar{b}_{1}+\bar{\beta}_{1}(l T)^{4}+\frac{1}{2}\left(\bar{b}_{2}+\bar{\beta}_{2}(l T)^{4}\right)\left(l \Lambda_{c}\right)^{2}+\frac{1}{4}\left(\bar{b}_{3}+\bar{\beta}_{3}(l T)^{4}\right)\left(l \Lambda_{c}\right)^{4}\right], \quad \bar{\beta}_{1}<0, \quad \bar{\beta}_{2}, \bar{\beta}_{3}>0,
\end{aligned}
$$

where $\hat{V}_{q \bar{q}}$ is dimensionless meson potential energy at low temperature. $\beta_{1}, \beta_{2}$ and $\beta_{3}$ are constant coefficients which are given by 
TABLE II. Numerical constant coefficients for thermal potential calculation.

\begin{tabular}{llll}
\hline \hline$\alpha_{1}=-0.119814$ & $\alpha_{2}=-0.274949$ & $\alpha_{3}=-0.330873$ & $\bar{a}_{4}=-0.116281$ \\
$\beta_{1}=-0.299535$ & $\beta_{2}=-0.671444$ & $\beta_{3}=-0.917564$ & \\
$\bar{\beta}_{1}=-3.23986$ & $\bar{\beta}_{2}=2.4604$ & $\bar{\beta}_{3}=1.94742$ & \\
\hline \hline
\end{tabular}

$$
\begin{aligned}
\bar{\beta}_{1}= & -\frac{27 \pi^{2} \Gamma\left(\frac{1}{4}\right) \Gamma\left(\frac{5}{4}\right)}{320 \Gamma\left(\frac{7}{4}\right)^{2}}, \\
\bar{\beta}_{2}= & \frac{\pi^{2}}{3828825 \Gamma\left(-\frac{1}{4}\right)^{7}}\left\{1 6 \Gamma ( \frac { 1 } { 4 } ) \left[312432120 \sqrt{2} \pi^{2}-75810735 \sqrt{2} \Gamma\left(\frac{1}{4}\right)^{4}\right.\right. \\
& -256 \sqrt{\pi} \Gamma\left(\frac{1}{4}\right)^{2}\left(51051_{3} F_{2}\left(\frac{3}{4}, \frac{5}{4}, \frac{3}{2} ; \frac{7}{4}, \frac{9}{4} ; 1\right)-303875_{3} F_{2}\left(\frac{3}{2}, \frac{7}{4}, \frac{9}{4} ; \frac{11}{4}, \frac{13}{4} ; 1\right)\right. \\
& +240975_{3} F_{2}\left(\frac{7}{4}, \frac{5}{2}, \frac{13}{4} ; \frac{15}{4}, \frac{17}{4} ; 1\right)-187425_{3} F_{2}\left(\frac{9}{4}, \frac{5}{2}, \frac{11}{4} ; \frac{15}{4}, \frac{17}{4} ; 1\right) \\
& \left.\left.\left.+9450_{3} F_{2}\left(\frac{11}{4}, \frac{13}{4}, \frac{7}{2} ; \frac{19}{4}, \frac{21}{4} ; 1\right)\right)\right]\right\} .
\end{aligned}
$$

$\bar{\beta}_{3}$ is not reported here due to its complex form. The numerical value of all the constant coefficients are summarized in Table II.

\section{APPENDIX B: HOLOGRAPHIC SUBREGION COMPLEXITY}

(a) Zero temperature: In Sec. VA 1, we obtain Eq. (41) for the dimensionless subregion volume $\tilde{V}_{\gamma_{A}}$ at zero temperature in the high energy limit $r_{c} \ll r_{*}$. Note that $\tilde{V}_{\gamma_{A}}$ is applied to achieve HSC using Eq. (12). Here we review the computations. Using Eq. (38) and up to $\left(r_{c} / r_{*}\right)^{4}$ and $\left(r_{h} / r_{*}\right)^{4}$ we get

$$
l\left(r_{*}\right)=\frac{2}{r_{*}} \sum_{n=0}^{\infty} \frac{\Gamma\left(n+\frac{1}{2}\right)}{\sqrt{\pi} \Gamma(n+1)} \int_{0}^{1} u^{6 n+3}\left[1+\frac{3}{2}(2 n+1)\left(1-u^{2}\right)\left(\frac{r_{c}}{r_{*}}\right)^{2}+\frac{9}{8}(2 n+1)^{2}\left(1-u^{2}\right)^{2}\left(\frac{r_{c}}{r_{*}}\right)^{4}\right] d u .
$$

Performing the above integral, we have

$$
l\left(r_{*}\right)=\frac{2}{r_{*}}\left[k_{1}+k_{2}\left(\frac{r_{c}}{r_{*}}\right)^{2}+k_{3}\left(\frac{r_{c}}{r_{*}}\right)^{4}\right], \quad k_{1}, k_{2}, k_{3}>0,
$$

where $k_{1}, k_{2}$ and $k_{3}$ are given by

$$
\begin{aligned}
k_{1} & \equiv \sum_{n=0}^{\infty} \frac{\Gamma\left(n+\frac{1}{2}\right)}{\sqrt{\pi} \Gamma(n+1)(6 n+4)}=\frac{\sqrt{\pi} \Gamma\left(\frac{5}{3}\right)}{4 \Gamma\left(\frac{7}{6}\right)}, \\
k_{2} & \equiv \sum_{n=0}^{\infty} \frac{\Gamma\left(n+\frac{1}{2}\right)(1+2 n)}{4 \sqrt{\pi} \Gamma(n+1)\left(2+5 n+3 n^{2}\right)}=\frac{1}{2}-\frac{\sqrt{\pi} \Gamma\left(\frac{5}{3}\right)}{8 \Gamma\left(\frac{7}{6}\right)}, \\
k_{3} & \equiv \sum_{n=0}^{\infty} \frac{3 \Gamma\left(n+\frac{1}{2}\right)(1+2 n)^{2}}{8 \sqrt{\pi} \Gamma(n+1)\left(8+26 n+27 n^{2}+9 n^{3}\right)} \\
& =\frac{3}{1120 \pi}\left\{189 \sqrt{\pi}\left[2 \Gamma\left(\frac{5}{3}\right) \Gamma\left(\frac{11}{6}\right)+\Gamma\left(\frac{7}{6}\right) \Gamma\left(\frac{7}{3}\right)\right]+8 \pi_{3} F_{2}\left(\frac{3}{2}, \frac{5}{3}, \frac{7}{3} ; \frac{8}{3}, \frac{10}{3} ; 1\right)-280 \pi\right\} .
\end{aligned}
$$

Solving (B2) for $r_{*}$ perturbatively we get

$$
r_{*}(l)=\frac{1}{l}\left(\bar{k}_{1}+\frac{\bar{k}_{2}}{2}(\sqrt{c} l)^{2}+\frac{\bar{k}_{3}}{4}(\sqrt{c} l)^{4}\right), \quad \bar{k}_{1}, \bar{k}_{2}>0, \quad \bar{k}_{3}<0,
$$


where $\bar{k}_{1}, \bar{k}_{2}$ and $\bar{k}_{3}$ are given by

$$
\begin{aligned}
& \bar{k}_{1}=\frac{\sqrt{\pi} \Gamma\left(\frac{5}{3}\right)}{2 \Gamma\left(\frac{7}{6}\right)}, \\
& \bar{k}_{2}=\frac{5 \times 2^{2 / 3} \pi \Gamma\left(\frac{4}{3}\right)+4 \Gamma\left(\frac{7}{6}\right)^{2}\left(-5+2{ }_{2} F_{1}\left(1, \frac{4}{3}, \frac{8}{3},-1\right)\right)}{5 \pi \Gamma\left(\frac{5}{3}\right)^{2}}, \\
& \bar{k}_{3}=\frac{1}{25515 \pi^{3 / 2} \Gamma\left(\frac{5}{3}\right)^{5} \Gamma\left(\frac{11}{6}\right)}\left\{5600 \times 2^{\frac{2}{3}} \sqrt{3} \pi^{\frac{5}{2}} \Gamma\left(\frac{1}{6}\right)-4725 \times 2^{1 / 3} \pi^{2} \Gamma\left(\frac{1}{3}\right)^{2}\right. \\
& +5670 \times 2^{2 / 3} \sqrt{\pi} \Gamma\left(\frac{1}{3}\right)^{2} \Gamma\left(\frac{7}{6}\right)^{3}+14 \Gamma\left(\frac{1}{6}\right)^{4}\left[75-4 \times 2^{2 / 3}{ }_{2} F_{1}\left(\frac{4}{3}, \frac{5}{3} ; \frac{8}{3} ;-1\right)^{2}\right] \\
& \left.+5 \times 2^{2 / 3} \pi \Gamma\left(\frac{1}{6}\right)^{2} \Gamma\left(\frac{1}{3}\right)\left[-1085+3_{3} F_{2}\left(\frac{3}{2}, \frac{5}{3}, \frac{7}{3} ; \frac{8}{3}, \frac{10}{3} ; 1\right)\right]\right\} .
\end{aligned}
$$

Using similar calculations for $V_{\gamma_{A}}$, Eq. (39) (and keeping up to $\left(r_{c} / r_{*}\right)^{4}$ and $\left.\left(r_{h} / r_{*}\right)^{4}\right)$ we obtain

$$
\begin{aligned}
\tilde{V}_{\gamma_{A}}\left(r_{*}\right)= & 2 L^{2} r_{*}^{2} \sum_{n=0}^{\infty} \frac{\Gamma\left(n+\frac{1}{2}\right)}{\sqrt{\pi} \Gamma(n+1)} \int_{\delta}^{1} d u\left\{\frac{1-u^{6 n+4}}{(6 n+4) u^{4}}\right. \\
& +\left[\frac{3}{2}(2 n+1)\left(\frac{u^{-4}}{6(3 n+2)(n+1)}+u^{6 n}\left(\frac{u^{2}}{6(2 n+1)}-\frac{1}{2(3 n+2)}\right)\right)+\frac{u^{-2}}{3 n+2}\left(1-u^{6 n+4}\right)\right]\left(\frac{r_{c}}{r_{*}}\right)^{2} \\
& +\left[3(2 n+1)\left(\frac{u^{-2}}{6(3 n+2)(n+1)}+u^{6 n+2}\left(\frac{u^{2}}{6(n+1)}-\frac{1}{2(3 n+2)}\right)\right)+\frac{1}{3 n+2}\left(1-u^{6 n+4}\right)\right. \\
& \left.\left.+\frac{9}{8}(2 n+1)^{2}\left(\frac{u^{-4}}{3(3 n+2)(3 n+4)(n+1)}+u^{6 n}\left(\frac{u^{2}}{3(n+1)}-\frac{u^{4}}{2(3 n+4)}-\frac{1}{2(3 n+2)}\right)\right)\right]\left(\frac{r_{c}}{r_{*}}\right)^{4}\right\} .
\end{aligned}
$$

Integrating (B6) and considering finite parts

$$
V_{\gamma_{A}}\left(r_{*}\right)=2 L^{2} r_{*}^{2}\left[w_{1}+w_{2}\left(\frac{r_{c}}{r_{*}}\right)^{2}+w_{3}\left(\frac{r_{c}}{r_{*}}\right)^{4}\right], \quad w_{1}, w_{2}, w_{3}<0
$$

where $w_{1}, w_{2}$ and $w_{3}$ are given by

$$
\begin{aligned}
& w_{1} \equiv-\sum_{n=0}^{\infty} \frac{\Gamma\left(n+\frac{1}{2}\right)}{3 \sqrt{\pi} \Gamma(n+1)(6 n+1)}=-\frac{\sqrt{\pi} \Gamma\left(\frac{7}{6}\right)}{3 \Gamma\left(\frac{2}{3}\right)}, \\
& w_{2} \equiv-\sum_{n=0}^{\infty} \frac{\Gamma\left(n+\frac{1}{2}\right)(3+14 n)}{\sqrt{\pi} \Gamma(n+1)\left(3+24 n+36 n^{2}\right)}=\frac{\pi}{4}-\frac{\sqrt{\pi} \Gamma\left(\frac{7}{6}\right)}{\Gamma\left(\frac{5}{3}\right)}-\frac{1}{9}{ }_{3} F_{2}\left(\frac{7}{6}, \frac{3}{2}, \frac{3}{2} ; \frac{13}{6}, \frac{5}{2} ; 1\right), \\
& w_{3} \equiv-\sum_{n=0}^{\infty} \frac{\Gamma\left(n+\frac{1}{2}\right)(1+2 n)}{\sqrt{\pi} \Gamma(n+1)\left(5+36 n+36 n^{2}\right)}=\frac{\sqrt{\pi}}{20}\left(\frac{\Gamma\left(\frac{11}{6}\right)}{\Gamma\left(\frac{4}{3}\right)}-\frac{5 \Gamma\left(\frac{7}{6}\right)}{\Gamma\left(\frac{2}{3}\right)}\right)-\frac{1}{77}{ }_{3} F_{2}\left(\frac{7}{6}, \frac{3}{2}, \frac{11}{6} ; \frac{13}{6}, \frac{17}{6} ; 1\right) .
\end{aligned}
$$

Using Eq. (B6) and substituting $r_{c}=\Lambda_{c} / \sqrt{2}$ we reach the final result

$$
\tilde{V}_{\gamma_{A}}\left(l \Lambda_{c}\right) \equiv \frac{V_{\gamma_{A}}\left(l, l \Lambda_{c}\right)}{L^{2} \Lambda_{c}^{2}}=\frac{1}{\left(l \Lambda_{c}\right)^{2}}\left(\bar{w}_{1}+\frac{\bar{w}_{2}}{2}\left(l \Lambda_{c}\right)^{2}+\frac{\bar{w}_{3}}{4}\left(l \Lambda_{c}\right)^{4}\right), \quad \bar{w}_{1}, \bar{w}_{2}, \bar{w}_{3}<0,
$$

where $\tilde{V}_{\gamma_{A}}$ is dimensionless subregion volume at zero temperature. $\bar{w}_{1}, \bar{w}_{2}$ and $\bar{w}_{3}$ are given by 


$$
\begin{aligned}
\bar{w}_{1}= & -\frac{\pi^{3 / 2} \Gamma\left(\frac{5}{3}\right)^{2}}{6 \Gamma\left(\frac{2}{3}\right) \Gamma\left(\frac{7}{6}\right)}, \\
\bar{w}_{2}= & \frac{1}{18}\left[9 \pi-\frac{2 \Gamma\left(\frac{1}{6}\right)^{2}}{\Gamma\left(\frac{2}{3}\right)^{2}}-\frac{7 \sqrt{\pi} \Gamma\left(\frac{1}{6}\right)}{\Gamma\left(\frac{2}{3}\right)}-4{ }_{3} F_{2}\left(\frac{7}{6}, \frac{3}{2}, \frac{3}{2} ; \frac{13}{6}, \frac{5}{2} ; 1\right)\right], \\
\bar{w}_{3}= & \frac{1}{1330560 \times 2^{1 / 3} \Gamma\left(\frac{2}{3}\right)^{5} \Gamma\left(\frac{8}{3}\right)}\left\{\left[-13305602^{2 / 3} \sqrt{\pi} \Gamma\left(\frac{1}{3}\right)^{3} \Gamma\left(\frac{7}{6}\right)^{3}\right.\right. \\
& -4065600 \sqrt{3} 2^{2 / 3} \pi^{5 / 2} \Gamma\left(\frac{1}{6}\right) \Gamma\left(\frac{4}{3}\right)+693 \pi^{2}\left[32002^{1 / 3} \Gamma\left(\frac{4}{3}\right)^{3}+243 \Gamma\left(\frac{5}{3}\right) \Gamma\left(\frac{8}{3}\right) \Gamma\left(\frac{11}{3}\right)\right] \\
& +3193344 \Gamma\left(\frac{7}{6}\right)^{4} \Gamma\left(\frac{4}{3}\right)\left[-325+16 \times 2^{2 / 3}{ }_{2} F_{1}\left(\frac{4}{3}, \frac{5}{3} ; \frac{8}{3} ;-1\right)^{2}\right] \\
& -256002^{2 / 3} \pi^{3}{ }_{3} F_{2}\left(\frac{7}{6}, \frac{3}{2}, \frac{11}{6} ; \frac{13}{6}, \frac{17}{6} ; 1\right)-42240 \pi \Gamma\left(\frac{1}{3}\right)^{2} \Gamma\left(\frac{7}{6}\right)^{2}\left(84{ }_{2} F_{1}\left(\frac{4}{3}, \frac{5}{3} ; \frac{8}{3} ;-1\right)\right. \\
& \left.\left.\left.+3 \times 2^{2 / 3}{ }_{3} F_{2}\left(\frac{3}{2}, \frac{5}{3}, \frac{7}{3} ; \frac{8}{3}, \frac{10}{3} ; 1\right)-1330 \times 2^{2 / 3}\right)\right]\right\} .
\end{aligned}
$$

All the constant coefficients are summarized in the Table III.

(b) Low temperature: In Sec. V B 1, Eq. (48) is reached for the dimensionless subregion volume $\hat{V}_{\gamma_{A}}$ at low temperature $l T \ll 1$ in the high energy limit $r_{c} \ll r_{*}$. HSC is obtained using Eq. (12). In the following we write the full calculation here. Expanding Eq. (45) up to $\left(r_{c} / r_{*}\right)^{4}$ and $\left(r_{h} / r_{*}\right)^{4}$ we have

$$
\begin{aligned}
l\left(r_{*}\right)= & \frac{2}{r_{*}} \sum_{n=0}^{\infty} \frac{\Gamma\left(n+\frac{1}{2}\right)}{\sqrt{\pi} \Gamma(n+1)} \int_{0}^{1} u^{6 n+3}\left\{1+\frac{1}{2} u^{6 n+4}\left(\frac{r_{h}}{r_{*}}\right)^{4}\right. \\
& +\left[\frac{3}{2}(2 n+1)\left(1-u^{2}\right)+\frac{3}{4}(2 n+1)\left(1-u^{2}\right) u^{6 n+4}\left(\frac{r_{h}}{r_{*}}\right)^{4}\right]\left(\frac{r_{c}}{r_{*}}\right)^{2} \\
& \left.+\left[\frac{9}{8}(2 n+1)^{2}\left(1-u^{2}\right)^{2}+\frac{9}{18}(2 n+1)^{2}\left(1-u^{2}\right)^{2} u^{6 n+4}\left(\frac{r_{h}}{r_{*}}\right)^{4}\right]\left(\frac{r_{c}}{r_{*}}\right)^{4}\right\} d u .
\end{aligned}
$$

Integrating Eq. (B11) we obtain

$$
l\left(r_{*}\right)=\frac{2}{r_{*}}\left[k_{1}+\kappa_{1}\left(\frac{r_{h}}{r_{*}}\right)^{4}+\left(k_{2}+\kappa_{2}\left(\frac{r_{h}}{r_{*}}\right)^{4}\right)\left(\frac{r_{c}}{r_{*}}\right)^{2}+\left(k_{3}+\kappa_{3}\left(\frac{r_{h}}{r_{*}}\right)^{4}\right)\left(\frac{r_{c}}{r_{*}}\right)^{4}\right], \quad \kappa_{1}, \kappa_{2}, \kappa_{3}>0
$$

where $\kappa_{1}, \kappa_{2}, \kappa_{3}$ are given by

TABLE III. Numerical constant coefficients for potential calculation.

\begin{tabular}{lll}
\hline \hline$k_{1}=0.431185$ & $k_{2}=0.284408$ & $k_{3}=0.180262$ \\
$\bar{k}_{1}=0.86237$ & $\bar{k}_{2}=0.764864$ & $\bar{k}_{3}=-0.704896$ \\
$w_{1}=-0.404775$ & $w_{2}=-1.45197$ & $w_{3}=-0.264624$ \\
$\bar{w}_{1}=-0.602048$ & $\bar{w}_{2}=-3.9719$ & $\bar{w}_{3}=-0.201038$ \\
\hline \hline
\end{tabular}




$$
\begin{aligned}
\kappa_{1} & \equiv \sum_{n=0}^{\infty} \frac{\Gamma\left(n+\frac{1}{2}\right)}{\sqrt{\pi} \Gamma(n+1)(12 n+16)}=\frac{\sqrt{\pi} \Gamma\left(\frac{7}{3}\right)}{16 \Gamma\left(\frac{11}{6}\right)}, \\
\kappa_{2} & \equiv \sum_{n=0}^{\infty} \frac{\Gamma\left(n+\frac{1}{2}\right)(6 n+3)}{\sqrt{\pi} \Gamma(n+1)\left(72 n^{2}+216 n+160\right)} \\
& =\frac{3}{2240}\left[\frac{72\left(3 \Gamma\left(\frac{13}{6}\right) \Gamma\left(\frac{7}{3}\right)-2 \Gamma\left(\frac{5}{6}\right) \Gamma\left(\frac{8}{3}\right)\right)}{\sqrt{\pi}}+5_{3} F_{2}\left(\frac{3}{2}, \frac{7}{3}, \frac{8}{3} ; \frac{10}{3}, \frac{11}{3} ; 1\right)\right], \\
\kappa_{3} & \equiv \sum_{n=0}^{\infty} \frac{\Gamma\left(n+\frac{1}{2}\right)(2 n+1)^{2}}{6 \sqrt{\pi} \Gamma(n+1)\left(9 n^{3}+45 n^{2}+74 n+40\right)} \\
& =1+\frac{9\left(95 \Gamma\left(\frac{13}{6}\right) \Gamma\left(\frac{7}{3}\right)-184 \Gamma\left(\frac{11}{6}\right) \Gamma\left(\frac{8}{3}\right)\right)}{700 \sqrt{\pi}}+\frac{1}{168}{ }_{3} F_{2}\left(\frac{3}{2}, \frac{7}{3}, \frac{8}{3} ; \frac{10}{3}, \frac{11}{3} ; 1\right) .
\end{aligned}
$$

Solving Eq. (B12) perturbatively we reach

$$
r_{*}(l)=\frac{1}{l}\left(\bar{k}_{1}+\frac{\bar{k}_{2}}{2}(\sqrt{c} l)^{2}+\frac{\bar{k}_{3}}{4}(\sqrt{c} l)^{4}+\bar{k}_{4}\left(r_{h} l\right)^{4}\right), \quad \bar{k}_{1}, \bar{k}_{2}, \bar{k}_{4}>0, \quad \bar{k}_{3}<0,
$$

where $\bar{k}_{4}$ is given by

$$
\bar{k}_{4}=\frac{2 \Gamma\left(\frac{7}{6}\right)^{4} \Gamma\left(\frac{7}{3}\right)}{\pi^{3 / 2} \Gamma\left(\frac{5}{3}\right)^{4} \Gamma\left(\frac{11}{6}\right)} .
$$

Similar calculations yield to an expression for $V_{\gamma_{A}}$ from Eq. (46)

$$
\begin{aligned}
V_{\gamma_{A}}\left(r_{*}\right)= & 2 V_{y, z} r_{*}^{2} \sum_{n=0}^{\infty} \frac{\Gamma\left(n+\frac{1}{2}\right)}{\sqrt{\pi} \Gamma(n+1)} \int_{\delta}^{1} d u\left\{\frac{1-u^{6 n+4}}{(6 n+4) u^{4}}+\left(\frac{1-u^{6 n+8}}{2(6 n+8) u^{4}}+\frac{1-u^{6 n+4}}{2(6 n+4)}\right)\left(\frac{r_{h}}{r_{*}}\right)^{4}\right. \\
& +\left[\frac{3}{2}(2 n+1)\left(\frac{u^{-4}}{6(3 n+2)(n+1)}+u^{6 n}\left(\frac{u^{2}}{6(n+1)}-\frac{1}{2(3 n+2)}\right)\right)+\frac{u^{-2}}{3 n+2}\left(1-u^{6 n+4}\right)\right. \\
& +\left(\frac { 3 } { 4 } ( 2 n + 1 ) \left(\frac{u^{-4}}{2(3 n+4)(3 n+5)}+u^{6 n}\left(\frac{(3 n+4) u^{6}}{3(3 n+5)(n+1)}+\frac{u^{4}}{(3 n+2)(3 n+4)}\right)\right.\right. \\
& \left.\left.\left.+\frac{1}{6(3 n+2)(n+1)}\right)+\frac{u^{-2}}{2(3 n+4)}\left(1-u^{6 n+8}\right)+\frac{u^{2}}{2(3 n+2)}\left(1-u^{6 n+4}\right)\right)\left(\frac{r_{h}}{r_{*}}\right)^{4}\right]\left(\frac{r_{c}}{r_{*}}\right)^{2} \\
& +\left[3(2 n+1)\left(\frac{u^{-2}}{6(3 n+2)(n+1)}+u^{6 n+2}\left(\frac{u^{2}}{6(n+1)}-\frac{1}{2(3 n+2)}\right)\right)+\frac{1}{3 n+2}\left(1-u^{6 n+4}\right)\right. \\
& +\frac{9}{8}(2 n+1)^{2}\left(\frac{u^{-4}}{3(3 n+2)(3 n+4)(n+1)}+u^{6 n}\left(\frac{u^{2}}{3(n+1)}-\frac{u^{4}}{2(3 n+4)}-\frac{1}{2(3 n+2)}\right)\right) \\
& +\left(\frac { 3 } { 2 } ( 2 n + 1 ) \left(\frac{u^{-2}}{2(3 n+4)(3 n+5)}+\frac{u^{2}}{6(3 n+2)(n+1)}+\frac{3 u^{6 n+6}(n+1)}{(3 n+2)(3 n+4)}\right.\right. \\
& \left.+\frac{u^{6 n+8}(3 n+4)}{3(3 n+5)(n+1)}\right)+\frac{9}{16}(2 n+1)^{2}\left(\frac{u^{-4}}{3(3 n+5)(3 n+4)(n+2)}+\frac{1}{(3 n+4)(3 n+2)(n+1)}\right. \\
& \left.+u^{6 n}\left(\frac{2(3 n+4) u^{6}}{3(3 n+5)(n+1) u^{6}}-\frac{3(n+1) u^{4}}{(3 n+2)(3 n+4)}-\frac{(6 n+5) u^{8}}{6(3 n+4)(n+2)}\right)\right)+\frac{u^{4}\left(1-u^{6 n+4}\right)}{6 n+4} \\
& \left.\left.\left.+\frac{1}{6 n+8}\left(1-u^{6 n+8}\right)\right)\left(\frac{r_{h}}{r_{*}}\right)^{4}\right]\left(\frac{r_{c}}{r_{*}}\right)^{4}\right\} d u .
\end{aligned}
$$

Integrating (B16) and considering finite parts yields 


$$
\begin{aligned}
V_{\gamma_{A}}\left(r_{*}\right)= & 2 V_{y, z} r_{*}^{2}\left[w_{1}+\omega_{1}\left(\frac{r_{h}}{r_{*}}\right)^{4}+\left(w_{2}+\omega_{2}\left(\frac{r_{h}}{r_{*}}\right)^{4}\right)\left(\frac{r_{c}}{r_{*}}\right)^{2}+\left(w_{3}+\omega_{3}\left(\frac{r_{h}}{r_{*}}\right)^{4}\right)\left(\frac{r_{c}}{r_{*}}\right)^{4}\right], \\
& \omega_{1}, \omega_{3}>0, \quad \omega_{2}<0,
\end{aligned}
$$

where $\omega_{1}, \omega_{3}$ and $\omega_{2}$ are given by

$$
\begin{aligned}
& \omega_{1} \equiv \sum_{n=0}^{\infty} \frac{\Gamma\left(n+\frac{1}{2}\right)}{\sqrt{\pi} \Gamma(n+1)(18 n+15)}=\frac{\sqrt{\pi} \Gamma\left(\frac{11}{6}\right)}{15 \Gamma\left(\frac{4}{3}\right)}, \\
& \omega_{2} \equiv-\sum_{n=0}^{\infty} \frac{\Gamma\left(n+\frac{1}{2}\right)}{\sqrt{\pi} \Gamma(n+1)(18 n+15)}=-\frac{\sqrt{\pi} \Gamma\left(\frac{11}{6}\right)}{15 \Gamma\left(\frac{4}{3}\right)}, \\
& \omega_{3} \equiv \sum_{n=0}^{\infty} \frac{\Gamma\left(n+\frac{1}{2}\right)(26 n+25)}{15 \sqrt{\pi} \Gamma(n+1)(2 n+3)(6 n+5)}=\frac{5 \pi}{24}-\frac{1}{6}{ }_{3} F_{2}\left(\frac{5}{6}, \frac{3}{2}, \frac{3}{2} ; \frac{11}{6}, \frac{5}{2} ; 1\right)+\frac{13}{825^{3}} F_{2}\left(\frac{3}{2}, \frac{11}{6}, \frac{5}{2} ; \frac{17}{6}, \frac{7}{2} ; 1\right) .
\end{aligned}
$$

Then by substituting (B14) in (B17) we get

$$
\begin{aligned}
\hat{V}_{\gamma_{A}}\left(l \Lambda_{c}, l T\right) \equiv & \frac{V_{\gamma_{A}}\left(l, l \Lambda_{c}, l T\right)}{L^{2} \Lambda_{c} T}=\frac{1}{l^{2} \Lambda_{c} T}\left(\bar{w}_{1}+\bar{\omega}_{1}(l T)^{4}+\frac{1}{2}\left(\bar{w}_{2}+\bar{\omega}_{2}(l T)^{4}\right)\left(l \Lambda_{c}\right)^{2}+\frac{1}{4}\left(\bar{w}_{3}+\bar{\omega}_{3}(l T)^{4}\right)\left(l \Lambda_{c}\right)^{4}\right), \\
& \bar{\omega}_{1}, \bar{\omega}_{2}<0, \quad \bar{\omega}_{3}>0,
\end{aligned}
$$

where $\hat{V}_{\gamma_{A}}$ is dimensionless subregion volume and $\bar{\omega}_{1}, \bar{\omega}_{2}$ and $\bar{\omega}_{3}$ are given by

$$
\begin{aligned}
\bar{\omega}_{1}= & \frac{\pi^{7 / 2} \Gamma\left(\frac{7}{6}\right)}{\sqrt{3} \Gamma\left(\frac{5}{3}\right)}-\frac{108 \times 2^{1 / 3} \pi^{2} \Gamma\left(\frac{7}{6}\right)^{6}}{5 \Gamma\left(\frac{2}{3}\right)^{3}}, \\
\bar{\omega}_{2}= & -\frac{2^{4 / 3} \pi^{3 / 2} \Gamma\left(\frac{7}{6}\right)^{2}}{9 \Gamma\left(\frac{2}{3}\right)^{7} \Gamma\left(\frac{8}{3}\right)}\left[10 \sqrt{3} \pi^{7 / 2}-9 \pi \Gamma\left(\frac{1}{3}\right)^{3} \Gamma\left(\frac{7}{6}\right)^{3}+162 \times 2^{1 / 3} \Gamma\left(\frac{1}{3}\right)^{2} \Gamma\left(\frac{7}{6}\right)^{5}\right], \\
\bar{\omega}_{3}= & \frac{\pi}{35925120 \times 2^{1 / 3} \Gamma\left(\frac{2}{3}\right)^{10} \Gamma\left(\frac{11}{6}\right) \Gamma\left(\frac{8}{3}\right)}\left\{27720000 \times 2^{1 / 3} \pi^{6} \Gamma\left(\frac{1}{6}\right)-115500 \pi^{3} \Gamma\left(\frac{1}{6}\right)^{7}-96096000 \sqrt{3} \pi^{11 / 2} \Gamma\left(\frac{1}{3}\right)^{2}\right. \\
& +59675 \times 2^{2 / 3} \pi \Gamma\left(\frac{1}{6}\right)^{7} \Gamma\left(\frac{1}{3}\right)^{3}+404157600 \times 2^{1 / 3} \pi^{2} \Gamma\left(\frac{1}{3}\right)^{4} \Gamma\left(\frac{7}{6}\right)^{5}-484989120 \times 2^{2 / 3} \sqrt{\pi} \Gamma\left(\frac{1}{3}\right)^{4} \Gamma\left(\frac{7}{6}\right)^{8} \\
& +154 \Gamma\left(\frac{1}{6}\right)^{9} \Gamma\left(\frac{1}{3}\right)^{2}\left[2^{8 / 3}{ }_{2} F_{1}\left(\frac{4}{3}, \frac{5}{3} ; \frac{8}{3} ;-1\right)^{2}-75\right] \\
& +46200 \pi^{7 / 2} \Gamma\left(\frac{1}{6}\right)^{4}\left[\sqrt{3}\left(8 F_{2} F_{1}\left(\frac{4}{3}, \frac{5}{3} ; \frac{8}{3} ;-1\right)+15 \times 2^{2 / 3}\right)-2^{8 / 3} \pi\right] \\
& +7200 \pi^{2} \Gamma\left(\frac{1}{6}\right)^{3} \Gamma\left(\frac{1}{3}\right)^{5}{ }_{3} F_{2}\left(\frac{7}{6}, \frac{3}{2}, \frac{11}{6} ; \frac{13}{6}, \frac{17}{6} ; 1\right)-110 \sqrt{3} \pi^{3 / 2} \Gamma\left(\frac{1}{6}\right)^{8} \Gamma\left(\frac{1}{3}\right){ }_{3} F_{2}\left(\frac{3}{2}, \frac{5}{3}, \frac{7}{3} ; \frac{8}{3}, \frac{10}{3} ; 1\right) \\
& +240 \times 2^{1 / 3} \pi^{9 / 2} \Gamma\left(\frac{1}{6}\right)^{2} \Gamma\left(\frac{1}{3}\right)\left[77000 \sqrt{3}+9625 \pi-12320 \sqrt{3}{ }_{2} F_{1}\left(1, \frac{4}{3} ; \frac{8}{3} ;-1\right)\right. \\
& \left.\left.-7700_{3} F_{2}\left(\frac{5}{6}, \frac{3}{2}, \frac{3}{2} ; \frac{11}{6}, \frac{5}{2} ; 1\right)-330 \sqrt{3}{ }_{3} F_{2}\left(\frac{3}{2}, \frac{5}{3}, \frac{7}{3} ; \frac{8}{3}, \frac{10}{3} ; 1\right)+728_{3} F_{2}\left(\frac{3}{2}, \frac{11}{6}, \frac{5}{2} ; \frac{17}{6}, \frac{7}{2} ; 1\right)\right]\right\} .
\end{aligned}
$$

All constant coefficients are reported in Table IV. 
TABLE IV. Numerical constant of thermal subregion complexity.

\begin{tabular}{llll}
\hline \hline$\kappa_{1}=0.140218$ & $\kappa_{2}=0.0806394$ & $\kappa_{3}=0.0480308$ & $\bar{k}_{4}=0.507061$ \\
$\omega_{1}=0.124472$ & $\omega_{2}=-0.124472$ & $\omega_{3}=0.245496$ & \\
$\bar{\omega}_{1}=-36.3574$ & $\bar{\omega}_{2}=-162.853$ & $\bar{\omega}_{3}=539.984$ & \\
\hline \hline
\end{tabular}

(c) High temperature: The constant coefficients corresponding to the Eqs. (59) and (66) in Sec. V B 2 are given as follows:

$$
\begin{aligned}
& A_{1}=\frac{\sqrt{\pi} \Gamma\left(\frac{1}{6}\right)}{18 \Gamma\left(\frac{2}{3}\right)}{ }_{5} F_{4}\left(-\frac{1}{4}, \frac{1}{12}, \frac{1}{6}, \frac{1}{2}, \frac{7}{12} ; \frac{1}{3}, \frac{1}{3}, \frac{2}{3}, \frac{3}{4} ; 1\right)-\frac{\sqrt{\pi} \Gamma\left(\frac{5}{6}\right)}{12 \Gamma\left(\frac{4}{3}\right)}{ }_{5} F_{4}\left(\frac{1}{12}, \frac{5}{12}, \frac{1}{2}, \frac{5}{6}, \frac{11}{12} ; \frac{2}{3}, \frac{13}{12}, \frac{4}{3}, \frac{3}{4} ; 1\right) \\
& -\frac{\pi}{160}{ }_{5} F_{4}\left(\frac{5}{12}, \frac{3}{4}, \frac{5}{6}, \frac{7}{6}, \frac{5}{4} ; 1, \frac{4}{3}, \frac{17}{12}, \frac{5}{3} ; 1\right) \\
& -{ }_{2} F_{1}\left(-\frac{3}{4}, \frac{1}{2} ; \frac{1}{4} ; 1\right)\left[\sum_{m=1}^{\infty}\left(\frac{\Gamma\left(m+\frac{1}{2}\right) \Gamma\left(\frac{2 m}{3}+\frac{2}{3}\right)}{18 \Gamma(m+1) \Gamma\left(\frac{2 m}{3}+\frac{7}{6}\right)}-\frac{1}{18 m}\right)+\frac{\sqrt{\pi} \Gamma\left(\frac{2}{3}\right)}{18 \Gamma\left(\frac{7}{6}\right)}\right] \\
& +\sum_{m=1}^{\infty}\left[\frac{\Gamma\left(m+\frac{1}{2}\right) \Gamma\left(\frac{2 m}{3}+\frac{1}{6}\right)}{18 \Gamma(m+1) \Gamma\left(\frac{2 m}{3}+\frac{2}{3}\right)}{ }_{6} F_{5}\left(-\frac{1}{4}, \frac{1}{6}, \frac{1}{2}, \frac{5}{6}, \frac{m}{3}+\frac{1}{12}, \frac{m}{3}+\frac{7}{12} ; \frac{1}{3}, \frac{2}{3}, \frac{3}{4}, \frac{m}{3}+\frac{1}{3}, \frac{m}{3}+\frac{5}{6} ; 1\right)\right. \\
& \left.-\frac{\sqrt{3}}{18 \sqrt{2}}{ }_{4} F_{3}\left(-\frac{1}{4}, \frac{1}{6}, \frac{1}{2}, \frac{5}{6} ; \frac{1}{3}, \frac{2}{3}, \frac{3}{4} ; 1\right) m^{-1}\right] \\
& -\sum_{m=1}^{\infty}\left[\frac{\Gamma\left(m+\frac{1}{2}\right) \Gamma\left(\frac{2 m}{3}+\frac{5}{6}\right)}{12 \Gamma(m+1) \Gamma\left(\frac{2 m}{3}+\frac{4}{3}\right)}{ }_{6} F_{5}\left(\frac{1}{12}, \frac{1}{2}, \frac{5}{6}, \frac{7}{6}, \frac{m}{3}+\frac{5}{12}, \frac{m}{3}+\frac{11}{12} ; \frac{2}{3}, \frac{13}{12}, \frac{4}{3}, \frac{m}{3}+\frac{2}{3}, \frac{m}{3}+\frac{7}{6} ; 1\right)\right. \\
& \left.-\frac{\sqrt{3} \Gamma\left(\frac{1}{6}\right) \Gamma\left(\frac{13}{12}\right) \Gamma\left(\frac{4}{3}\right)}{2 \sqrt{2} \Gamma\left(\frac{1}{12}\right) \Gamma\left(\frac{1}{3}\right) \Gamma\left(\frac{7}{6}\right)}{ }_{4} F_{3}\left(\frac{1}{12}, \frac{1}{2}, \frac{5}{6}, \frac{7}{6} ; \frac{2}{3}, \frac{13}{12}, \frac{4}{3} ; 1\right) m^{-1}\right] \\
& -\sum_{m=1}^{\infty}\left[\frac{\Gamma\left(m+\frac{1}{2}\right) \Gamma\left(\frac{2 m}{3}+\frac{3}{2}\right)}{80 \Gamma(m+1) \Gamma\left(\frac{2 m}{3}+2\right)}{ }_{6} F_{5}\left(\frac{5}{12}, \frac{5}{6}, \frac{7}{6}, \frac{3}{2}, \frac{m}{3}+\frac{3}{4}, \frac{m}{3}+\frac{5}{4} ; \frac{4}{3}, \frac{17}{12}, \frac{5}{3}, \frac{m}{3}+1, \frac{m}{3}+\frac{3}{2} ; 1\right)\right. \\
& \left.-\frac{9 \sqrt{3} \Gamma\left(\frac{1}{6}\right) \Gamma\left(\frac{4}{3}\right) \Gamma\left(\frac{17}{12}\right) \Gamma\left(\frac{5}{3}\right)}{400 \sqrt{2} \Gamma\left(\frac{1}{3}\right) \Gamma\left(\frac{5}{12}\right) \Gamma\left(\frac{2}{3}\right) \Gamma\left(\frac{7}{6}\right)}{ }_{4} F_{3}\left(\frac{5}{12}, \frac{5}{6}, \frac{7}{6}, \frac{3}{2} ; \frac{4}{3}, \frac{17}{12}, \frac{5}{3} ; 1\right) m^{-1}\right],
\end{aligned}
$$

$$
\begin{aligned}
A_{2}= & \frac{\sqrt{3} \Gamma\left(\frac{1}{6}\right) \Gamma\left(\frac{13}{12}\right) \Gamma\left(\frac{4}{3}\right)}{2 \sqrt{2} \Gamma\left(\frac{1}{12}\right) \Gamma\left(\frac{1}{3}\right) \Gamma\left(\frac{7}{6}\right)}{ }_{4} F_{3}\left(\frac{1}{12}, \frac{1}{2}, \frac{5}{6}, \frac{7}{6} ; \frac{2}{3}, \frac{13}{12}, \frac{4}{3} ; 1\right) \\
& +\frac{9 \sqrt{3} \Gamma\left(\frac{1}{6}\right) \Gamma\left(\frac{4}{3}\right) \Gamma\left(\frac{17}{12}\right) \Gamma\left(\frac{5}{3}\right)}{400 \sqrt{2} \Gamma\left(\frac{1}{3}\right) \Gamma\left(\frac{5}{12}\right) \Gamma\left(\frac{2}{3}\right) \Gamma\left(\frac{7}{6}\right)}{ }_{4} F_{3}\left(\frac{5}{12}, \frac{5}{6}, \frac{7}{6}, \frac{3}{2} ; \frac{4}{3}, \frac{17}{12}, \frac{5}{3} ; 1\right) \\
& -\frac{\sqrt{3}}{18 \sqrt{2}}{ }_{4} F_{3}\left(-\frac{1}{4}, \frac{1}{6}, \frac{1}{2}, \frac{5}{6} ; \frac{1}{3}, \frac{2}{3}, \frac{3}{4} ; 1\right)+\frac{\sqrt{3}}{18 \sqrt{2}}{ }_{2} F_{1}\left(-\frac{3}{4}, \frac{1}{2} ; \frac{1}{4} ; 1\right) .
\end{aligned}
$$




$$
\begin{aligned}
& B_{1}=\sum_{n=0}^{\infty} \sum_{m=0}^{\infty}\left[\frac{3(2 n+1) \Gamma\left(n+\frac{1}{2}\right) \Gamma\left(m+\frac{1}{2}\right)}{4 \pi(3 n+2 m+3)(6 n+4 m+3) \Gamma(m+1) \Gamma(n+1)}{ }_{2} F_{1}\left(\frac{1}{2}, m+\frac{3 n}{2}+\frac{3}{4} ; m+\frac{3 n}{2}+\frac{7}{4} ; 1\right)\right. \\
& \left.-\frac{3(2 n+1) \Gamma\left(n+\frac{1}{2}\right) \Gamma\left(m+\frac{1}{2}\right)}{4 \pi(3 n+2 m+2)(6 n+4 m+1) \Gamma(n+1) \Gamma(m+1)}{ }_{2} F_{1}\left(\frac{1}{2}, m+\frac{3 n}{2}+\frac{1}{4} ; m+\frac{3 n}{2}+\frac{5}{4} ; 1\right)\right] \\
& -\frac{\pi}{3}{ }_{5} F_{4}\left(-\frac{1}{12}, \frac{1}{6}, \frac{1}{4}, \frac{3}{4}, \frac{5}{6} ; \frac{1}{3}, \frac{2}{3}, \frac{11}{12}, 1 ; 1\right)+\frac{\sqrt{\pi} \Gamma\left(\frac{13}{6}\right)}{21 \Gamma\left(\frac{5}{3}\right)}{ }_{5} F_{4}\left(\frac{1}{4}, \frac{1}{2}, \frac{7}{12}, \frac{13}{12}, \frac{7}{6} ; \frac{2}{3}, \frac{5}{4}, \frac{4}{3}, \frac{4}{3} ; 1\right) \\
& +\frac{3 \sqrt{\pi} \Gamma\left(\frac{17}{6}\right)}{308 \Gamma\left(\frac{7}{3}\right)}{ }_{5} F_{4}\left(\frac{7}{12}, \frac{5}{6}, \frac{11}{12}, \frac{17}{12}, \frac{3}{2} ; \frac{4}{3}, \frac{19}{12}, \frac{5}{3}, \frac{5}{3} ; 1\right) \\
& -\sum_{m=1}^{\infty}\left(\frac{\Gamma\left(m+\frac{1}{2}\right) \Gamma\left(\frac{2 m}{3}+\frac{3}{2}\right)}{(4 m+3)(4 m+7)(4 m+11) \Gamma(m+1) \Gamma\left(\frac{2 m}{3}+1\right)}(154+144 m)\right) \\
& \times{ }_{6} F_{5}\left(-\frac{1}{12}, \frac{1}{6}, \frac{1}{2}, \frac{5}{6}, \frac{m}{3}+\frac{1}{4}, \frac{m}{3}+\frac{3}{4} ; \frac{1}{3}, \frac{2}{3}, \frac{11}{12}, \frac{m}{3}+\frac{1}{2}, \frac{m}{3}+1 ; 1\right) \\
& +\sum_{m=1}^{\infty}\left(\frac{\Gamma\left(m+\frac{1}{2}\right) \Gamma\left(\frac{2 m}{3}+\frac{13}{6}\right)}{(4 m+3)(4 m+7)(4 m+11) \Gamma(m+1) \Gamma\left(\frac{2 m}{3}+\frac{5}{3}\right)}\left(11+\frac{56}{3} m\right)\right) \\
& \times{ }_{6} F_{5}\left(\frac{1}{4}, \frac{1}{2}, \frac{5}{6}, \frac{7}{6}, \frac{m}{3}+\frac{7}{12}, \frac{m}{3}+\frac{13}{12} ; \frac{2}{3}, \frac{5}{4}, \frac{4}{3}, \frac{m}{3}+\frac{5}{6}, \frac{m}{3}+\frac{4}{3} ; 1\right) \\
& +\sum_{m=1}^{\infty}\left(\frac{\Gamma\left(m+\frac{1}{2}\right) \Gamma\left(\frac{2 m}{3}+\frac{17}{6}\right)}{(4 m+3)(4 m+7)(4 m+11) \Gamma(m+1) \Gamma\left(\frac{2 m}{3}+\frac{7}{3}\right)}\left(\frac{9}{4}+\frac{30}{7} m\right)\right) \\
& \times{ }_{6} F_{5}\left(\frac{7}{12}, \frac{5}{6}, \frac{7}{6}, \frac{3}{2}, \frac{m}{3}+\frac{11}{12}, \frac{m}{3}+\frac{17}{12} ; \frac{4}{3}, \frac{19}{12}, \frac{5}{3}, \frac{m}{3}+\frac{7}{6}, \frac{m}{3}+\frac{5}{3} ; 1\right) \\
& -\sum_{m=1}^{\infty}\left(\frac{32 \Gamma\left(m+\frac{1}{2}\right) \Gamma\left(\frac{2 m}{3}+\frac{3}{2}\right) m^{2}}{(4 m+3)(4 m+7)(4 m+11) \Gamma(m+1) \Gamma\left(\frac{2 m}{3}+1\right)}\right. \\
& \times{ }_{6} F_{5}\left(-\frac{1}{12}, \frac{1}{6}, \frac{1}{2}, \frac{5}{6}, \frac{m}{3}+\frac{1}{4}, \frac{m}{3}+\frac{3}{4} ; \frac{1}{3}, \frac{2}{3}, \frac{11}{12}, \frac{m}{3}+\frac{1}{2}, \frac{m}{3}+1 ; 1\right) \\
& \left.-\frac{1}{\sqrt{6}}{ }_{4} F_{3}\left(-\frac{1}{12}, \frac{1}{6}, \frac{1}{2}, \frac{5}{6} ; \frac{1}{3}, \frac{2}{3}, \frac{11}{12} ; 1\right) m^{-1}\right) \\
& +\sum_{m=1}^{\infty}\left(\frac{16 \Gamma\left(m+\frac{1}{2}\right) \Gamma\left(\frac{2 m}{3}+\frac{13}{6}\right) m^{2}}{3(4 m+3)(4 m+7)(4 m+11) \Gamma(m+1) \Gamma\left(\frac{2 m}{3}+\frac{5}{3}\right)}\right. \\
& \times{ }_{6} F_{5}\left(\frac{1}{4}, \frac{1}{2}, \frac{5}{6}, \frac{7}{6}, \frac{m}{3}+\frac{7}{12}, \frac{m}{3}+\frac{13}{12} ; \frac{2}{3}, \frac{5}{4}, \frac{4}{3}, \frac{m}{3}+\frac{5}{6}, \frac{m}{3}+\frac{4}{3} ; 1\right) \\
& \left.-\frac{\sqrt{2} \Gamma\left(\frac{1}{6}\right) \Gamma\left(\frac{5}{4}\right) \Gamma\left(\frac{4}{3}\right)}{6 \sqrt{3} \Gamma\left(\frac{1}{4}\right) \Gamma\left(\frac{1}{3}\right) \Gamma\left(\frac{7}{6}\right)}{ }_{4} F_{3}\left(\frac{1}{4}, \frac{1}{2}, \frac{5}{6}, \frac{7}{6} ; \frac{2}{3}, \frac{5}{4}, \frac{4}{3} ; 1\right) m^{-1}\right) \\
& +\sum_{m=1}^{\infty}\left(\frac{12 \Gamma\left(m+\frac{1}{2}\right) \Gamma\left(\frac{2 m}{3}+\frac{17}{6}\right) m^{2}}{7(4 m+3)(4 m+7)(4 m+11) \Gamma(m+1) \Gamma\left(\frac{2 m}{3}+\frac{7}{3}\right)}\right. \\
& \times{ }_{6} F_{5}\left(\frac{7}{12}, \frac{5}{6}, \frac{7}{6}, \frac{3}{2}, \frac{m}{3}+\frac{11}{12}, \frac{m}{3}+\frac{17}{12} ; \frac{4}{3}, \frac{19}{12}, \frac{5}{3}, \frac{m}{3}+\frac{7}{6}, \frac{m}{3}+\frac{5}{3} ; 1\right) \\
& \left.-\frac{27 \sqrt{2} \Gamma\left(\frac{1}{6}\right) \Gamma\left(\frac{4}{3}\right) \Gamma\left(\frac{19}{12}\right) \Gamma\left(\frac{5}{3}\right)}{784 \sqrt{3} \Gamma\left(\frac{1}{3}\right) \Gamma\left(\frac{7}{12}\right) \Gamma\left(\frac{2}{3}\right) \Gamma\left(\frac{7}{6}\right)} F_{3}\left(\frac{7}{12}, \frac{5}{6}, \frac{7}{6}, \frac{3}{2} ; \frac{4}{3}, \frac{19}{12}, \frac{5}{3} ; 1\right) m^{-1}\right),
\end{aligned}
$$




$$
\begin{aligned}
B_{2}= & \frac{1}{\sqrt{6}}{ }_{4} F_{3}\left(-\frac{1}{12}, \frac{1}{6}, \frac{1}{2}, \frac{5}{6} ; \frac{1}{3}, \frac{2}{3}, \frac{11}{12} ; 1\right)-\frac{\sqrt{2} \Gamma\left(\frac{1}{6}\right) \Gamma\left(\frac{5}{4}\right) \Gamma\left(\frac{4}{3}\right)}{6 \sqrt{3} \Gamma\left(\frac{1}{4}\right) \Gamma\left(\frac{1}{3}\right) \Gamma\left(\frac{7}{6}\right)} F_{4} F_{3}\left(\frac{1}{4}, \frac{1}{2}, \frac{5}{6}, \frac{7}{6} ; \frac{2}{3}, \frac{5}{4}, \frac{4}{3} ; 1\right) \\
& -\frac{27 \sqrt{2} \Gamma\left(\frac{1}{6}\right) \Gamma\left(\frac{4}{3}\right) \Gamma\left(\frac{19}{12}\right) \Gamma\left(\frac{5}{3}\right)}{784 \sqrt{3} \Gamma\left(\frac{1}{3}\right) \Gamma\left(\frac{7}{12}\right) \Gamma\left(\frac{2}{3}\right) \Gamma\left(\frac{7}{6}\right)} F_{4} F_{3}\left(\frac{7}{12}, \frac{5}{6}, \frac{7}{6}, \frac{3}{2} ; \frac{4}{3}, \frac{19}{12}, \frac{5}{3} ; 1\right) .
\end{aligned}
$$

Now for finding $\ln (4 \epsilon)$ as a function of $l$ at leading and sub-leading order, we follow below procedure: At high temperature, we reach the finite expression for $l$ up to $\left(r_{c} / r_{*}\right)^{2}$, Eq. (52). Here, we consider zero order of $\left(r_{c} / r_{*}\right)$ and do sum over $n$, we obtain

$$
l\left(r_{*}\right)=\frac{1}{r_{*}} \sum_{m=0}^{\infty} \frac{\Gamma\left(m+\frac{1}{2}\right) \Gamma\left(\frac{2 m}{3}+\frac{2}{3}\right)}{3 \Gamma(m+1) \Gamma\left(\frac{2 m}{3}+\frac{7}{6}\right)}\left(\frac{r_{h}}{r_{*}}\right)^{4 m} .
$$

For large $m$, this series goes as $\sim m^{-1}\left(r_{h} / r_{*}\right)^{4 m}$ and hence it diverges for $r_{*}=r_{h}$. By isolating the divergence part of the series (B25), we get

$$
l\left(r_{*}\right)=\frac{1}{r_{*}}\left[\frac{\sqrt{\pi} \Gamma\left(\frac{2}{3}\right)}{3 \Gamma\left(\frac{7}{6}\right)}+\sum_{m=1}^{\infty}\left(\frac{\Gamma\left(m+\frac{1}{2}\right) \Gamma\left(\frac{2 m}{3}+\frac{2}{3}\right)}{3 \Gamma(m+1) \Gamma\left(\frac{2 m}{3}+\frac{7}{6}\right)}-\frac{1}{\sqrt{6} m}\right)\left(\frac{r_{h}}{r_{*}}\right)^{4 m}-\frac{1}{\sqrt{6}} \ln \left[1-\left(\frac{r_{h}}{r_{*}}\right)^{4}\right]\right] .
$$

The convergence of the above infinite series can be obviously seen for $r_{*}=r_{h}$. We write $r_{*}=r_{h}(1+\epsilon)$, following the fact that at high temperature $\epsilon \ll 1$, we obtain

$$
\ln (4 \epsilon)=-\sqrt{6} r_{h} l+\frac{\sqrt{6 \pi} \Gamma\left(\frac{2}{3}\right)}{3 \Gamma\left(\frac{7}{6}\right)}+\sum_{m=1}^{\infty}\left(\frac{\sqrt{6} \Gamma\left(m+\frac{1}{2}\right) \Gamma\left(\frac{2 m}{3}+\frac{2}{3}\right)}{3 \Gamma(m+1) \Gamma\left(\frac{2 m}{3}+\frac{7}{6}\right)}-\frac{1}{m}\right)+\mathcal{O}(\epsilon) .
$$

Now, we consider the second order of $\left(r_{c} / r_{*}\right)$ and sum over $n$ for zero order of $\left(r_{c} / r_{*}\right)$ and over $m$ for second order of $\left(r_{c} / r_{*}\right)$, we get

$$
\begin{aligned}
l\left(r_{*}\right)= & \frac{1}{r_{*}} \sum_{m=0}^{\infty} \frac{\Gamma\left(m+\frac{1}{2}\right) \Gamma\left(\frac{2 m}{3}+\frac{2}{3}\right)}{3 \Gamma(m+1) \Gamma\left(\frac{2 m}{3}+\frac{7}{6}\right)}\left(\frac{r_{h}}{r_{*}}\right)^{4 m}+\frac{1}{r_{*}} \sum_{n=0}^{\infty}\left[\frac{3(2 n+1) \Gamma\left(n+\frac{1}{2}\right)}{2(3 n+2) \sqrt{\pi} \Gamma(n+1)}{ }_{2} F_{1}\left(\frac{1}{2}, \frac{3 n}{2}+1 ; \frac{3 n}{2}+2 ;\left(\frac{r_{h}}{r_{*}}\right)^{4}\right)\right. \\
& \left.-\frac{(2 n+1) \Gamma\left(n+\frac{1}{2}\right)}{2(n+1) \sqrt{\pi} \Gamma(n+1)}{ }_{2} F_{1}\left(\frac{1}{2}, \frac{3 n}{2}+\frac{3}{2} ; \frac{3 n}{2}+\frac{5}{2} ;\left(\frac{r_{h}}{r_{*}}\right)^{4}\right)\right]\left(\frac{r_{c}}{r_{*}}\right)^{2} .
\end{aligned}
$$

The investigation of convergence of the first sum, summation over $m$, is similar to Eq. (B25) which was studied before. For large $n$, the behavior of two terms corresponding to the second sum, summation over $n$, is the same and hence it is a convergent series. We write $r_{*}=r_{h}(1+\epsilon)$, knowing that at high temperature $\epsilon \ll 1$, we get

$$
\begin{aligned}
\ln (4 \epsilon)= & -\sqrt{6} r_{h} l+\frac{\sqrt{6 \pi} \Gamma\left(\frac{2}{3}\right)}{3 \Gamma\left(\frac{7}{6}\right)}+\sum_{m=1}^{\infty}\left(\frac{\sqrt{6} \Gamma\left(m+\frac{1}{2}\right) \Gamma\left(\frac{2 m}{3}+\frac{2}{3}\right)}{3 \Gamma(m+1) \Gamma\left(\frac{2 m}{3}+\frac{7}{6}\right)}-\frac{1}{m}\right) \\
& +\sqrt{6} \sum_{n=0}^{\infty}\left[\frac{3(2 n+1) \Gamma\left(n+\frac{1}{2}\right)}{2(3 n+2) \sqrt{\pi} \Gamma(n+1)}{ }_{2} F_{1}\left(\frac{1}{2}, \frac{3 n}{2}+1 ; \frac{3 n}{2}+2 ; 1\right)\right. \\
& \left.-\frac{(2 n+1) \Gamma\left(n+\frac{1}{2}\right)}{2(n+1) \sqrt{\pi} \Gamma(n+1)}{ }_{2} F_{1}\left(\frac{1}{2}, \frac{3 n}{2}+\frac{3}{2} ; \frac{3 n}{2}+\frac{5}{2} ; 1\right)\right]\left(\frac{r_{c}}{r_{h}}\right)^{2}+\mathcal{O}(\epsilon) .
\end{aligned}
$$

As expected when $r_{c}=0$, we have (B27). 
[1] J. M. Maldacena, The large N limit of superconformal field theories and supergravity, Int. J. Theor. Phys. 38, 1113 (1999).

[2] J. Casalderrey-Solana, H. Liu, D. Mateos, K. Rajagopal, and U. A. Wiedemann, Gauge/String Duality, Hot QCD and Heavy Ion Collisions (Cambridge University Press, Cambridge, England, 2014).

[3] M. Attems, J. Casalderrey-Solana, D. Mateos, I. Papadimitriou, D. Santos-Olivan, C. F. Sopuerta, M. Triana, and M. Zilhao, Thermodyamics, transport and relaxation in nonconformal theories, J. High Energy Phys. 10 (2016) 155.

[4] K. G. Wilson, Confinement of quarks, Phys. Rev. D 10, 2445 (1974).

[5] J. Sonnenschein and A. Loewy, On the supergravity evaluation of wilson loop correlators in confining theories, J. High Energy Phys. 01 (2000) 042.

[6] J. Erdmenger, K. Ghoroku, R. Meyer, and L. Papadimitriou, Holographic cosmological backgrounds, wilson loop (De) confimement and dilaton singularities, Fortschr. Phys. 60, 991 (2012).

[7] M. Ammon and J. Erdmenger, Gauge/Gravity Duality: Foundations and Applications (Cambridge University Press, Cambridge, England, 2015).

[8] M. Ali-Akbari and M. Lezgi, Holographic QCD, entanglement entropy, and critical temperature, Phys. Rev. D 96, 086014 (2017).

[9] I. R. Klebanov, D. Kutasov, and A. Murugan, Entanglement as a probe of confinement, Nucl. Phys. B796, 274 (2008).

[10] G. Vidal, J. I. Latorre, E. Rico, and A. Kitaev, Entanglement in Quantum Critical Phenomena, Phys. Rev. Lett. 90, 227902 (2003).

[11] I. Bah, A. Faraggi, L. A. Pando Zayas, and C. A. TerreroEscalante, Holographic entanglement entropy and phase transitions at finite temperature, Int. J. Mod. Phys. A 24, 2703 (2009).

[12] J. Knaute and B. Kampfer, Holographic entanglement entropy in the QCD phase diagram with a critical point, Phys. Rev. D 96, 106003 (2017).

[13] D. Dudal and S. Mahapatra, Confining gauge theories and holographic entanglement entropy with a magnetic field, J. High Energy Phys. 04 (2017) 106003.

[14] L. Bombelli, R. K. Koul, J. Lee, and R. D. Sorkin, A quantum source of entropy for black holes, Phys. Rev. D 34, 373 (1986).

[15] M. Srednicki, Entropy and Area, Phys. Rev.Lett. 71,666(1993).

[16] S. Ryu and T. Takayanagi, Holographic Derivation of Entanglement Entropy from AdS/CFT, Phys. Rev. Lett. 96, 181602 (2006).

[17] J. Watrous, Quantum computational complexity, arXiv: quant-ph/0804.3401.

[18] S. Aaronson, The complexity of quantum states and transformations: From quantum money to black holes, arXiv: quant-ph/0804.3401.
[19] D. Stanford and L. Susskind, Complexity and shock wave geometries, Phys. Rev. D 90, 126007 (2014).

[20] A. R. Brown, D. A. Roberts, L. Susskind, B. Swingle, and Y. Zhao, Complexity, action, and black holes, Phys. Rev. D 93, 086006 (2016).

[21] A. R. Brown, D. A. Roberts, L. Susskind, B. Swingle, and Y. Zhao, Holographic Complexity Equals Bulk Action?, Phys. Rev. Lett. 116, 191301 (2016).

[22] M. Alishahiha, Holographic complexity, Phys. Rev. D 92 , 126009 (2015).

[23] D. Carmi, R. C. Myers, and P. Rath, Comments on holographic complexity, J. High Energy Phys. 03 (2017) 118.

[24] B. Chen, W.-M. Li, R.-Q. Yang, C.-Y. Zhang, and S.-J. Zhang, Holographic subregion complexity under a thermal quench, J. High Energy Phys. 07 (2018) 034.

[25] S. J. Zhang, Complexity and phase transitions in a holographic QCD model, Nucl. Phys. B929, 243 (2018).

[26] M. Lezgi and M. Ali-Akbari, A note on holographic subregion complexity and QCD phase transition, Phys. Rev. D 101, 026022 (2020).

[27] O. Ben-Ami and D. Carmi, On volumes of subregions in holography and complexity, J. High Energy Phys. 11 (2016) 129.

[28] S. J. Zhang, Subregion complexity in holographic thermalization with dS boundary, Eur. Phys. J. C 79, 715 (2019).

[29] P. Roy and T. Sarkar, On subregion holographic complexity and renormalization group flows, Phys. Rev. D 97, 086018 (2018).

[30] M. Asadi, On volume subregion complexity in nonconformal theories, Eur. Phys. J. C 80, 681 (2020).

[31] W. Fischler and S. Kundu, Strongly coupled gauge theories: High and low temperature behavior of nonlocal observables, J. High Energy Phys. 05 (2013) 098.

[32] P. Roy and T. Sarkar, Note on subregion holographic complexity, Phys. Rev. D 96, 026022 (2017).

[33] H. Ebrahim and G. M. Nafisi, Holographic mutual information and critical exponents of the strongly coupled plasma, Phys. Rev. D 102, 106007 (2020).

[34] O. Andreev and V. I. Zakharov, Heavy-quark potentials and AdS/QCD, Phys. Rev. D 74, 025023 (2006).

[35] O. Andreev and V. I. Zakharov, The spatial string tension, thermal phase transition, and AdS/QCD, Phys. Lett. B 645, 437 (2007).

[36] J. M. Maldacena, Wilson Loops in Large N Field Theories, Phys. Rev. Lett. 80, 4859 (1998).

[37] S. Ryu and T. Takayanagi, Aspects of holographic entanglement entropy, J. High Energy Phys. 08 (2006) 045.

[38] E. Eichten, K. Gottfried, T. Konoshita, K. D. Lane, and T.-M. Yan, Charmonium: The model, Phys. Rev. D 17, 3090 (1978); Charmonium: Comparison with experiment, Phys. Rev. D 21, 203 (1980). 